\title{
SMOOTH REPRESENTATIONS AND SHEAVES
}

\author{
U.JANNSEN, M.ROVINSKY
}

To Pierre Deligne on the occasion of his 65th birthday

\begin{abstract}
The paper is concerned with 'geometrization' of smooth (i.e. with open stabilizers) representations of the automorphism group of universal domains, and with the properties of 'geometric' representations of such groups. As an application, we calculate the cohomology groups of several classes of smooth representations of the automorphism group of an algebraically closed extension of infinite transcendence degree of an algebraically closed field.
\end{abstract}

Our motivation comes from an attempt to understand interplay between geometry ( $K$-groups) and topology (cohomology) of algebraic varieties, expressed in the motivic conjectures, through representations of certain 'big' totally disconnected topological groups, cf. [R4]. However, in this paper we reverse the point of view and study 'geometrization' of such representations.

0.1. Notations and terminology. Let $k$ be a characteristic zero field, $F \mid k$ be an algebraically closed extension of countable transcendence degree, and $G=G_{F \mid k}$ be the group of field automorphisms of $F$ leaving $k$ fixed. Let $B$ be the collection of the pointwise stabilizers of the finite subsets in $F$, i.e., the subgroups of type $G_{F \mid L}$ for subfields $L$ of $F \mid k$ of finite type.

We consider $G$ as a totally disconnected topological group with base of open subgroups $B$. These notions are recalled in Appendix A.

For a totally disconnected group $H$ an $H$-set is called smooth if the stabilizers of all its elements are open.

Let $E$ be a field endowed with a smooth $H$-action (by field automorphisms).

Denote by $\mathcal{S}_{H}(E)$ the category of smooth $E$-semilinear representations of $H$, i.e., $E$-vector spaces $V$ endowed with a smooth additive action of $H$ such that $h(e v)=h e \cdot h v$ for any $e \in E$, $h \in H, v \in V$. We set $\mathcal{S} m_{H}:=\mathcal{S} m_{H}(\mathbb{Q})$. Here the $H$-action on $\mathbb{Q}$ is trivial, which is the only possibility in the case $H=G$.

The full subcategory $\mathcal{I}_{G}$ of $\mathcal{S} m_{G}$, whose objects are 'homotopy invariant', is defined on $\mathrm{p}$.

For a variety $X$ over a field we denote by $C H^{q}(X)$ the (Chow) group of cycles of codimension $q$ modulo rational equivalence, i.e., modulo divisors of rational functions on subvarieties of codimension $q-1$.

For an abelian group $A$ we set $A_{\mathbb{Q}}:=A \otimes \mathbb{Q}$.

For a unitary commutative ring $A$ and $A$-algebras $A_{1}$ and $A_{2}$ denote by $\left\{A_{1} \stackrel{/ A}{\longrightarrow} A_{2}\right\}$ the set of unitary $A$-homomorphisms $A_{1} \stackrel{/ A}{\longrightarrow} A_{2}$. (If $A_{1}$ is a field then the homomorphisms are injectives, so the notation will be $\left\{A_{1} \stackrel{/ A}{\hookrightarrow} A_{2}\right\}$.) If $S$ is a set then $A[S]$ denotes the free $A$-module with basis $S$.

An algebraic closure of a field $L$ is denoted by $\bar{L}$, if $L$ is a subfield in $F$ then $\bar{L}$ denotes the algebraic closure in $F$.

0.2. Context and content. We are interested in interplay between algebraic geometry and representation theory of $G$. Various geometric categories are linked to the representations of $G$ by fully

Key words and phrases. Automorphism groups of fields, smooth representations, Grothendieck topologies. 
faithful functors. An example of such full embedding is given in Appendix B more examples are in [R1, Theorem 1.1, Proposition 4.3]. It is explained in [R2, Corollary 7.9] that it is especially important for geometric applications to describe the irreducible smooth representations of $G$ (or at least 'homotopy invariant' ones among them, cf. p 5 below).

It is well-known that for any totally disconnected group $H$ the smooth (i.e. with open stabilizers) $H$-sets and their $H$-equivariant maps form a topos, cf. Appendix A.1

In this paper, we introduce two sites, called the dominant and the Hartogs topologies, respectively. They are quite different from that described in $\$$.11 in the case of $H=G$.

In a way similar to Galois-Grothendieck correspondence, Lemma 1.1 describes the smooth $G$-sets as the sheaves in the dominant topology. Then we apply the standard sheaf-theoretic techniques to the study of the cohomology of smooth $G$-modules. Roughly, one of our results (Corollary 3.8) states the acyclicity of the smooth $G$-module corresponding to the dominant sheafification of a presheaf on the category of smooth $k$-varieties.

We deduce in Corollary 3.9 that for $E=\mathbb{Q}$ or $E=F$ (with the standard $G$-action) the category $\mathcal{S m}_{G}(E)$ is of infinite cohomological dimension, which is quite expectable.

However, there are 'too many' smooth representations of $G$, cf. Appendix A.5. For this reason, instead of studying all smooth representations of $G$, one can try to restrict the category of considered representations. More particularly, one can look for the irreducible $F$-semilinear representations of $G$ containing 'homotopy invariant' representations, expecting that there are 'very few' of them.

To explore this option we introduce in $\$ 4$ a functor from the category of smooth representations of $G$ to the category of sheaves in the Hartogs topology. From the point of vue of this functor, the 'homotopy invariant' representations 'look as local systems' (Proposition 4.1), and the 'interesting' $F$-semilinear representations of $G$ are 'globally generated' (Remark 1 after Lemma 4.2).

Homological machinery could be applied to several classes of questions. We mention just four examples.

(1) The acyclicity of some classes of representations is often crucial, e.g. for the knowledge of the structure of some co-induced representations, cf. $\$ 0.3$.

(2) One knows several cases when the Hom-groups in the triangulated category of mixed motives turn out to be related (dual) to corresponding Ext-groups in the category of 'homotopy invariant' representations of $G$, cf. [R1]. So one of the problems is to find other Ext-groups in the category of 'homotopy invariant' (and more generally, smooth) representations of $G$ and compare them with the conjectural values of corresponding Ext-groups in the conjectural category of mixed motives.

(3) There are some conjectures coming from geometry: on the semi-simplicity of the graded quotients of the level filtration $N_{\bullet}$, cf. [R1, Conjecture 6.9], on the splitting of the filtration $\mathcal{F}^{\bullet}$, ibid, etc.

(4) It is desirable to enlarge the category of 'homotopy invariant' representations and relate it to the category of effective mixed motives, so that in particular, this bigger category would contain objects like $F^{\times}$and $\mathbb{Q}$ was still a projective object.

0.3. From modules over Hecke algebras to modules over subquotient groups of $G$. In the standard type of semi-simplicity or irreducibility criteria of $G$-modules of Appendix A.3, it would be natural to replace the semi-simplicity or the irreducibility conditions for the modules over the Hecke algebras by the corresponding conditions on representations of the groups $G_{F^{\prime} \mid k}$ for the algebraically closed extensions $F^{\prime} \mid k$ in $F$ of finite transcendence degree. This is how the semi-simplicity of the $G$-module $B^{q}\left(X_{F}\right)$ is established in [R1, Proposition 3.8]. (Here $B^{q}\left(X_{F}\right)$ is 
the Chow group $C H^{q}\left(X_{F}\right)_{\mathbb{Q}}$ modulo the 'numerical equivalence over $k$ '.) More precisely, this is based on the following

Lemma. Let $W \in \mathcal{S} m_{G}$. If $\operatorname{Hom}_{G}\left(Z^{\operatorname{dim} X}\left(k(X) \otimes_{k} F\right), W\right)=\operatorname{Hom}_{G}\left(C H_{0}\left(X_{F}\right), W\right)$ for any smooth proper variety $X$ over $k$ then $W$ is semi-simple if and only if the $G_{F^{\prime} \mid k}$-module $W^{G_{F \mid F^{\prime}}}$ is semi-simple for any algebraically closed $F^{\prime}$ of finite transcendence degree over $k$.

Proof. Clearly, $W^{G_{F \mid L^{\prime}}}=W^{G_{F \mid L}}$ for any purely transcendental extension $L^{\prime} \mid L$ in $F \mid k$, i.e., $W \in \mathcal{I}_{G}$, cf. p 5 and [R1].

By the semi-simplicity criterion of Appendix A.3, Remark 1, p 13, the $G$-module $W$ is semisimple if and only if for any $L^{\prime} \mid L$ as above with $L$ of finite type over $k$ and $F$ algebraic over $L^{\prime}$ the $\mathcal{H}_{G_{F \mid L^{\prime}}}$-module $W^{G_{F \mid L^{\prime}}}$ is semi-simple. Here $\mathcal{H}_{G_{F \mid L^{\prime}}}:=h_{L^{\prime}} \mathbb{D}_{\mathbb{Q}}(G) h_{L^{\prime}} \supseteq\left\langle h_{L^{\prime}} \sigma h_{L^{\prime}} \mid \sigma \in G\right\rangle_{\mathbb{Q}}$ is the Hecke algebra and $h_{L^{\prime}}$ is the Haar measure on $G_{F \mid L^{\prime}}$.

As $W$ is a quotient of a direct sum of objects of type $C H_{0}\left(X_{F}\right)_{\mathbb{Q}}$, the action

$$
\mathbb{D}_{\mathbb{Q}}(G) \otimes W^{G_{F \mid L}} \longrightarrow \mathbb{Q}\left[G / G_{F \mid L}\right] \otimes W^{G_{F \mid L}} \longrightarrow W
$$

factors through $C H_{0}\left(Y_{F}\right)_{\mathbb{Q}} \otimes W^{G_{F \mid L}} \longrightarrow W$, where $Y$ is a smooth proper model of the extension $L \mid k$, cf. [R1, Proposition 3.6].

Thus, the action of $\mathcal{H}_{G_{F \mid L^{\prime}}}$ factors through $C H_{0}\left(Y_{k(Y)}\right)_{\mathbb{Q}}=h_{L^{\prime}} C H_{0}\left(Y_{F}\right)_{\mathbb{Q}}$.

In other words, the action of the Hecke algebra $\mathcal{H}_{G_{F \mid L^{\prime}}}(G)$ on $W^{G_{F \mid L}}$ is determined by the action of the Hecke algebra $\mathcal{H}_{G_{\bar{L} \mid L}}\left(G_{\bar{L} \mid k}\right)$, so the semi-simplicity of the $\mathcal{H}_{G_{F \mid L^{\prime}}}(G)$-module $W^{G_{F \mid L}}$ is equivalent to its semi-simplicity as a $\mathcal{H}_{G_{\bar{L} \mid L}}\left(G_{\bar{L} \mid k}\right)$-module.

The following lemma reduces the general semi-simplicity problem to an acyclicity question.

Lemma. Let $\mathcal{H}$ be a subcategory in $\mathcal{S} m_{G}$ closed under taking subobjects and $F^{\prime} \mid k$ be an algebraically closed extension in $F$ of a finite transcendence degree $q$. The following conditions on the subcategory $\mathcal{H}$ and on $F^{\prime}$ are equivalent.

(1) For any $W \in \mathcal{H}$ any $G_{F^{\prime} \mid k}$-submodule $U \subseteq W^{G_{F \mid F^{\prime}}}$ coincides with the $G_{F^{\prime} \mid k}$-submodule of

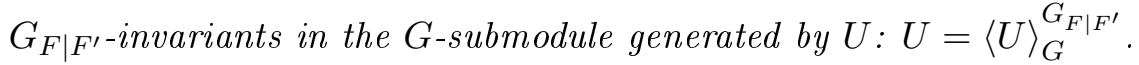

(2) For any $W \in \mathcal{H}$, any finitely generated field extension $L \mid k$ of transcendence degree $q$ and any surjection $\mathbb{Q}[\{L \stackrel{/ k}{\longleftrightarrow} F\}]^{N} \longrightarrow W$ in $\mathcal{S} m_{G}$ induces a surjection of $G_{F \mid F^{\prime} \text {-invariants }}$ $\mathbb{Q}\left[\left\{L \stackrel{/ k}{\hookrightarrow} F^{\prime}\right\}\right]^{N} \longrightarrow W^{G_{F \mid F^{\prime}}}$ in $\mathcal{S} m_{G_{F^{\prime} \mid k}}$, where $F^{\prime}$ is algebraic over $L$.

(3) For any finitely generated field extension $L \mid k$ of transcendence degree $q$ and for any $Q \subseteq$ $\mathbb{Q}[\{L \stackrel{/ k}{\hookrightarrow} F\}]^{N}$ such that the quotient belongs to $\mathcal{H}$, one has $H_{\mathcal{S} m_{G}}^{1}\left(G_{F \mid F^{\prime}}, Q\right)=0$.

Proof. (2) and (3) are equivalent by Corollary [3.9, since the restriction of $\mathbb{Q}[\{L \stackrel{/ k}{\hookrightarrow} F\}]$ to $G_{F \mid F^{\prime}}$ is canonically isomorphic to $\mathbb{Q}\left[\left\{L \otimes_{k} F^{\prime} \stackrel{/ F^{\prime}}{\longrightarrow} F\right\}\right]=\bigoplus_{x \in \operatorname{Spec}\left(L \otimes_{k} F^{\prime}\right)} \mathbb{Q}\left[\left\{F^{\prime}(x) \stackrel{/ F^{\prime}}{\hookrightarrow} F\right\}\right]$.

(2) $\Rightarrow(11)$. If $U \neq\langle U\rangle_{G}^{G_{F \mid F^{\prime}}}$ then there are elements $u_{1}, \ldots, u_{N} \in U, a_{i j} \in \mathbb{Q}$, and $g_{i j} \in G$ such that $\sum_{1 \leq i \leq N, j} a_{i j} g_{i j} u_{i} \in W^{G_{F \mid F^{\prime}}} \backslash U$. Replace $W$ by the $G$-span of $u_{1}, \ldots, u_{N}$, and $U$ by the $G_{F^{\prime} \mid k^{-}}$span of $u_{1}, \ldots, u_{N}$. Then there is a surjection $\mathbb{Q}[\{L \stackrel{/ k}{\hookrightarrow} F\}]^{N} \longrightarrow W, e_{i} \mapsto u_{i}$, where $L \mid k$ is an extension in $F^{\prime}$ of finite type such that $G_{F \mid L}$ is contained in the common stabilizer of the elements $u_{1}, \ldots, u_{N}$. Then (2) implies the surjectivity of $\mathbb{Q}\left[\left\{L \stackrel{/ k}{\hookrightarrow} F^{\prime}\right\}\right]^{N} \longrightarrow W^{G_{F \mid F^{\prime}}}$. As it factors through $U$, we get $U=W^{G_{F \mid F^{\prime}}}$, as required. 


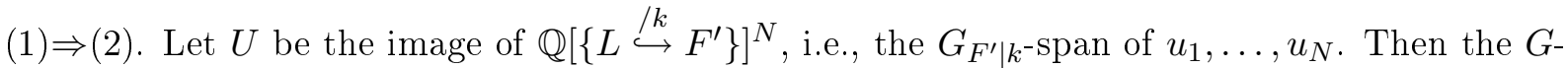
span of $u_{1}, \ldots, u_{N}$ coincides with $W$. It follows from (1) that $U=W^{G_{F \mid F^{\prime}}}$, i.e., the homomorphism $\mathbb{Q}\left[\left\{L \stackrel{/ k}{\longleftrightarrow} F^{\prime}\right\}\right]^{N} \longrightarrow W^{G_{F \mid F^{\prime}}}$ is surjective.

Examples. 1. Let $X$ be an irreducible $k$-variety with the function field $L$ embedded into $F$ and $Q \subseteq \mathbb{Q}[\{L \stackrel{/ k}{\hookrightarrow} F\}]$ be a $G$-submodule such that $\mathbb{Q}[\{L \stackrel{/ k}{\hookrightarrow} F\}] / Q$ is a quotient of $C H_{0}\left(X_{F}\right)_{\mathbb{Q}}$. Then it follows from the moving lemma of [R1] and from Corollary 3.9 that $H_{\mathcal{S} m_{G}}^{1}\left(G_{F \mid F^{\prime}}, Q\right)=0$.

2. ('Local acyclicity'). Suppose that the whole category $\mathcal{S} m_{G}$ satisfies the equivalent conditions of the previous lemma. Then for any finitely generated smooth representation $W$ of $G$ there exists an open subgroup $U \subset G$ such that $H_{\mathcal{S} m_{G}}^{>0}\left(U^{\prime}, W\right)=0$ for any open subgroup $U^{\prime} \subseteq U$ of type $G_{F \mid L}$. (Indeed, a choice of $N$ generators of $W$ determines a surjection $\pi: \mathbb{Q}[\{K \stackrel{/ k}{\hookrightarrow} F\}]^{N} \longrightarrow W$ for a field extension of finite type $K \mid k$. Then for any field extension $L \mid K$ of finite type both $\mathbb{Q}[\{K \stackrel{/ k}{\hookrightarrow} F\}]^{N}$ and the kernel of $\pi$ are acyclic with respect to $H_{\mathcal{S} m_{G}}^{>0}\left(G_{F \mid L},-\right)$.

An application, we have in mind is, e.g., to the semi-simplicity of the modules of regular differential forms and of similar representations. This is related to the coincidence of homological and numerical equivalence relations on algebraic cycles. Some known results are collected in Appendix C,

\section{The DOMinANT TOPOLOGY}

Consider the category $\mathfrak{D} m_{k}$ of smooth $k$-morphisms of smooth $k$-schemes endowed with the pretopology, where the covers of an object $X$ are dominant morphisms to $X$.

Given a presheaf $\mathcal{F}$ on $\mathfrak{D} m_{k}$ one can extend it to (the spectra of) the filtered unions $\mathcal{O}=$ $\lim _{A \longrightarrow} A$ of finitely generated smooth $k$-subalgebras $A$ by $\mathcal{F}(\mathcal{O}):=\lim _{A} \mathcal{F}(\operatorname{Spec}(A))$. Clearly, this is independent of presentation of $\mathcal{O}$ as a filtered union. Our main examples of $\mathcal{O}$ will be fields.

Lemma 1.1. The functor $\mathfrak{W}: \mathcal{F} \mapsto \mathcal{F}(F)$ is an equivalence between the category of sheaves of sets (resp., of abelian groups etc) on $\mathfrak{D} m_{k}$ and the category of smooth $G$-sets (resp., of $G$-modules etc).

Proof. Conversely, to define a quasi-inverse functor $\mathfrak{F}$ from the category of smooth $G$-sets to the category of sheaves of sets on $\mathfrak{D} m_{k}$, fix an embedding over $k$ into $F$ of the function field of each connected component of each object of $\mathfrak{D} m_{k}$. Then to each $G$-set $W$ and an object $\coprod_{\alpha} U_{\alpha}$, for some collection $\left(U_{\alpha}\right)_{\alpha}$ of irreducible objects of $\mathfrak{D} m_{k}$ we associate $\prod_{\alpha} W^{G_{F \mid k\left(U_{\alpha}\right)}}$. Note, that each homomorphism $K \stackrel{\tau}{\hookrightarrow} L$ of subfields in $F$ of finite type over $k$ extends to an element $\widetilde{\tau} \in G$, and the double coset $G_{F \mid L} \widetilde{\tau} G_{F \mid K}$ depends only on $\tau$, so the morphism $W^{G_{F \mid K}} \stackrel{\tau}{\longrightarrow} W^{G_{F \mid L}}$ is independent of a particular choice of $\widetilde{\tau}$. Then to a morphism $\coprod_{\beta \in T} V_{\beta} \stackrel{f}{\longrightarrow} \coprod_{\alpha \in S} U_{\alpha}$, given by a collection $\left(k\left(U_{f(\beta)}\right) \stackrel{\tau_{\beta}}{\hookrightarrow}\right.$ $\left.k\left(V_{\beta}\right)\right)_{\beta}$ we associate the morphism

$$
\prod_{\alpha \in S} W^{G_{F \mid k\left(U_{\alpha}\right)}} \stackrel{\left(\tau_{\beta}\right)}{\longrightarrow} \prod_{\alpha \in S} \prod_{\beta \in T_{\alpha}} W^{G_{F \mid k\left(V_{\beta}\right)}}=\prod_{\beta \in T} W^{G_{F \mid k\left(V_{\beta}\right)}} .
$$

The sheaf condition means that (i) the morphism (1) is injective and (ii) it is the equalizer of

$$
\prod_{\alpha \in S} \prod_{\beta \in T_{\alpha}} W^{G_{F \mid k\left(V_{\beta}\right)}} \rightrightarrows \prod_{\alpha \in S} \prod_{\beta, \beta^{\prime} \in T_{\alpha}} \prod_{x} W^{G_{F \mid k(x)}},
$$


where $x$ runs over irreducible components of $V_{\beta} \times_{U_{\alpha}} V_{\beta^{\prime}}$, for any cover $\coprod_{\beta \in T} V_{\beta} \stackrel{f}{\longrightarrow} \coprod_{\alpha \in S} U_{\alpha}$. The injectivity of (11) is evident. To verify the condition (ii), we apply [R1, Proposition 2.14]: if $L_{1}$ and $L_{2}$ are subextensions in $F \mid k$ of finite type such that the intersection of algebraic closures in $F$ of $L_{1}$ and $L_{2}$ is algebraic over $L_{1} \bigcap L_{2}$ then the subgroups $G_{F \mid L_{1}}$ and $G_{F \mid L_{2}}$ in $G$ generate $G_{F \mid L_{1}} \cap L_{2}$. Namely, if $\left(w_{\beta}\right)_{\beta \in T}$ belongs to the equalizer then $w_{\beta} \in W^{G_{F \mid k\left(U_{f(\beta)}\right)}}$ and $w_{\beta}=w_{\beta^{\prime}}$ if $f(\beta)=f\left(\beta^{\prime}\right)$, which means that $\left(w_{\beta}\right)_{\beta \in T}$ belongs to the image of (1).

Evidently, $\mathfrak{W} \mathfrak{F} \cong I d$. To check that $\mathfrak{F} \mathfrak{W} \cong I d$, it suffices to verify that $\mathcal{F}(U)=(\mathfrak{W} \mathcal{F})^{G_{F \mid k(U)}}$. But this is exactly the sheaf condition for the covers of $U$ by single connected elements.

\section{2. 'ACYCLICITY' OF CERTAIN RESTRICTIONS OF $G$-MODULES}

Proposition 2.1. Suppose that $F^{\prime}$ is an extension of $k$ in $F$ of infinite transcendence degree.

Then $H_{\mathcal{S} m_{G}}^{>0}\left(G_{F \mid \overline{F^{\prime}}}, W\right)=H_{\mathcal{S} m_{G}}^{>0}\left(G_{F \mid F^{\prime}}, W \otimes \mathbb{Q}\right)=0$ for any smooth $G$-module $W$.

Proof. Let $I^{\bullet}$ be an injective resolution of $W$ in the category $\mathcal{S} m_{G}$. As the functor $H^{0}\left(G_{F \mid \overline{F^{\prime}}},-\right)$ : $\mathcal{S} m_{G} \longrightarrow \mathcal{S} m_{G_{\overline{F^{\prime}} \mid k}}$ is an equivalence of categories (cf. [R1]), it is exact. This gives the vanishing of $H_{\mathcal{S} m_{G}}^{>0}\left(G_{F \mid \overline{F^{\prime}}}, W\right):=H^{>0}\left(\left(I^{\bullet}\right)^{G_{F \mid \overline{F^{\prime}}}}\right)$.

As the functor $H^{0}\left(G_{F \mid F^{\prime}},-\right): \mathcal{S} m_{G}(\mathbb{Q}) \longrightarrow \mathcal{A} b$ is a composition of exact functors $H^{0}\left(G_{F \mid \overline{F^{\prime}}},-\right)$ : $\mathcal{S} m_{G}(\mathbb{Q}) \longrightarrow \mathcal{S} m_{G_{\overline{F^{\prime}} \mid k}}(\mathbb{Q})$ and $H^{0}\left(G_{\overline{F^{\prime}} \mid F^{\prime}},-\right): \mathcal{S} m_{G_{\overline{F^{\prime}} \mid k}}(\mathbb{Q}) \longrightarrow \mathcal{A} b$, it is exact itself, and thus, $H_{\mathcal{S} m_{G}}^{>0}\left(G_{F \mid F^{\prime}}, W \otimes \mathbb{Q}\right):=H^{>0}\left(\left(I^{\bullet} \otimes \mathbb{Q}\right)^{G_{F \mid F^{\prime}}}\right)=0$.

\section{3. ČECH COHOMOLOGY}

Lemma 3.1. Let $S \stackrel{\pi}{\longrightarrow} T$ be a map of sets. For each $q \geq 0$, let $\stackrel{\circ}{S_{T}^{q}} \subseteq{ }_{\circ} \times_{T}^{q} S$ be a subset of the fibre product such that restriction to $\stackrel{\circ}{S}_{T}^{q}$ of each projection factors through $S_{T}^{q-1}$ and for any finite subset $U$ in $\stackrel{\circ}{S_{T}^{q}}$ over any element of $T$ (i.e. with $\#(\pi(U))=1$ ) there is $u \in S$ such that $\{u\} \times U \subseteq S_{T}^{q+1}$. Then the natural complex (where $d_{i}$ are alternating sums of projections) $\mathbb{Q}\left[S_{T}^{0}\right] \stackrel{d_{1}}{\longleftarrow} \mathbb{Q}\left[S_{T}^{1}\right] \stackrel{d_{2}}{\longleftarrow}$ $\mathbb{Q}\left[\stackrel{\circ}{S_{T}^{2}}\right] \stackrel{d_{3}}{\longleftarrow} \mathbb{Q}\left[\stackrel{\circ}{S_{T}^{3}}\right] \stackrel{d_{4}}{\longleftarrow} \ldots$ is acyclic.

Proof. Let $\alpha=\sum_{i} a_{i}\left(s_{1 i}, \ldots, s_{q i}\right) \in \operatorname{ker} d_{q}$. Then

$$
d_{q+1}: \sum_{i} a_{i}\left(u_{i}, s_{1 i}, \ldots, s_{q i}\right) \mapsto \sum_{i} a_{i}\left(s_{1 i}, \ldots, s_{q i}\right)+\sum_{i} \sum_{j=1}^{q}(-1)^{j} a_{i}\left(u_{i}, s_{1 i}, \ldots, \widehat{s_{j i}}, \ldots, s_{q i}\right)=\alpha
$$

for any collection $\left\{u_{i}\right\}_{i}$ such that $\left(u_{i}, s_{1 i}, \ldots, s_{q i}\right) \in \stackrel{\circ}{q+1}$ for all $i$ and $u_{i}$ depends only on the projection of $s_{1 i}$ to $T$.

Denote by $\mathcal{I}_{G}$ the full subcategory of $\mathcal{S} m_{G}$, whose objects $W$ satisfy $W^{G_{F \mid L^{\prime}}}=W^{G_{F \mid L}}$ for any purely transcendental extension $L^{\prime} \mid L$ in $F \mid k$. More on this can be found in [R1]. The equivalence of categories of Lemma 1.1 restricts to an equivalence of categories between $\mathcal{I}_{G}$ and the category of $\mathbb{A}^{1}$-invariant sheaves on $\mathfrak{D} m_{k}: \mathcal{F}(X)=\mathcal{F}\left(X \times \mathbb{A}^{1}\right)$ for any smooth $k$-variety $X$. This is why the objects of $\mathcal{I}_{G}$ are called 'homotopy invariant' representations. 
Let $\mathcal{I}: S m_{G} \longrightarrow \mathcal{I}_{G}$ be the left adjoint of the inclusion functor $\mathcal{I}_{G} \hookrightarrow \mathcal{S} m_{G}$ and $C_{k(Y)}:=$ $\mathcal{I} \mathbb{Q}[\{k(Y) \stackrel{/ k}{\hookrightarrow} F\}]$ for any irreducible $k$-variety $Y$. In the case of smooth proper $Y$, the object $C_{k(Y)}$ is related to the Chow group of zero-cycles on $Y$ : there is a natural surjection $C_{k(Y)} \longrightarrow C H_{0}\left(Y_{F}\right)_{\mathbb{Q}}$.

Lemma 3.2. The complex $\check{C}_{\bullet}^{L(Y) \mid L}:=\left(\cdots \rightarrow \mathbb{Q}\left[\left\{L\left(Y^{2}\right) \stackrel{/ k}{\hookrightarrow} F\right\}\right] \rightarrow \mathbb{Q}[\{L(Y) \stackrel{/ k}{\hookrightarrow} F\}] \rightarrow \mathbb{Q}[\{L \stackrel{/ k}{\hookrightarrow}\right.$ $F\}] \rightarrow 0)$ is acyclic for any field extension $L \mid k$ and any $L$-variety $Y$. If $Y$ is a smooth proper $k$ variety then the complexes $\cdots \rightarrow C_{k\left(Y^{2}\right)} \rightarrow C_{k(Y)} \rightarrow \mathbb{Q} \rightarrow 0$ and $\cdots \rightarrow C H_{0}\left(Y^{2}\right)_{\mathbb{Q}} \rightarrow C H_{0}(Y)_{\mathbb{Q}} \rightarrow$ $\mathbb{Q} \rightarrow 0$ are also acyclic.

Proof. The acyclicity of $\check{C}_{\bullet}^{L(Y) \mid L}$ follows from Lemma 3.1 applied to $T=\{L \stackrel{/ k}{\hookrightarrow} F\}$ and $S_{T}^{q}=$ $\left\{L\left(Y^{q}\right) \stackrel{/ k}{\hookrightarrow} F\right\}$.

Let $\alpha$ be an element of either $C_{k\left(Y^{q}\right)}$, or of $C H_{0}\left(Y^{q}\right)_{\mathbb{Q}}$ annihilated by the differential. As the morphisms $\mathbb{Q}\left[\left\{k\left(Y^{q}\right) \stackrel{/ k}{\hookrightarrow} F\right\}\right] \rightarrow C_{k\left(Y^{q}\right)} \rightarrow C H_{0}\left(Y_{F}^{q}\right)_{\mathbb{Q}}$ are surjective, $\alpha$ is represented by $\sum_{i} a_{i}\left(s_{1 i}, \ldots, s_{q i}\right)$. Let $u \in Y(F)$ be a generic point in generic position with respect to all $s_{j i}$, and $\beta$ be the image of $\sum_{i} a_{i}\left(u, s_{1 i}, \ldots, s_{q i}\right)$ in $C_{k\left(Y^{q}\right)}$, or in $C H_{0}\left(Y^{q}\right)_{\mathbb{Q}}$. Then $\partial \beta=\alpha+$ $\sum_{i} a_{i} \sum_{j=1}^{q}(-1)^{j}\left[\left(u, s_{1 i}, \ldots, \widehat{s_{j i}}, \ldots, s_{q i}\right)\right]$.

It remains to show that $\sum_{i} a_{i} \sum_{j=1}^{q}(-1)^{j}\left[\left(u, s_{1 i}, \ldots, \widehat{s_{j i}}, \ldots, s_{q i}\right)\right]=0$, which is clear in the case of $\mathrm{CH}_{0}$.

For the case of $C_{k(Y} \bullet$ note that for any smooth representation $W$ the kernel of the surjection $W \rightarrow \mathcal{I} W$ is generated by the elements $\sigma w-w$ for $w \in W^{G_{F \mid L^{\prime}}}, \sigma \in G_{F \mid L}$ and all purely transcendental extensions $L^{\prime} \mid L$. Let $W=\mathbb{Q}\left[\left\{k\left(Y^{q-1}\right) \stackrel{/ k}{\hookrightarrow} F\right\}\right]$. Then $\mathcal{I} W=C_{k\left(Y^{q-1}\right)}$ and $\sum_{i} a_{i} \sum_{j=1}^{q}(-1)^{j}\left(s_{1 i}, \ldots, \widehat{s_{j i}}, \ldots, s_{q i}\right)=\sum_{s}\left(\sigma_{s} w_{s}-w_{s}\right)$, where $w_{s} \in W^{G_{F \mid L_{s}^{\prime}}}, \sigma_{s} \in G_{F \mid L_{s}}$ and extensions $L_{s}^{\prime} \mid L_{s}$ are purely transcendental and of finite type over $k$.

Let $u: k(Y) \stackrel{/ k}{\hookrightarrow} F$ be a generic point of $Y$ in generic position with respect to all $L_{s}^{\prime}$ and $\sigma_{s}\left(L_{s}^{\prime}\right)$. Then there exists a collection of $\sigma_{s}^{\prime} \in G_{F \mid L_{s}}$ such that $\sigma_{s}^{\prime} w_{s}=\sigma_{s} w_{s}$ and $\sigma_{s}^{\prime} u=u$, so $\sum_{i} a_{i} \sum_{j=1}^{q}(-1)^{j}\left(u, s_{1 i}, \ldots, \widehat{s_{j i}}, \ldots, s_{q i}\right)=\sum_{s}\left(\sigma_{s}^{\prime}\left(u \otimes w_{s}\right)-u \otimes w_{s}\right) \in \mathbb{Q}\left[\left\{k\left(Y^{q}\right) \stackrel{/ k}{\hookrightarrow} F\right\}\right] \subset$ $\mathbb{Q}[\{k(Y) \stackrel{/ k}{\hookrightarrow} F\}] \otimes W$, i.e. $\sum_{i} a_{i} \sum_{j=1}^{q}(-1)^{j}\left[\left(u, s_{1 i}, \ldots, \widehat{s_{j i}}, \ldots, s_{q i}\right)\right]=0$ in $C_{k\left(Y^{q}\right)}$, since $u \otimes w_{s}$ is fixed by $G_{F \mid L_{s}^{\prime} u(k(Y))}, L_{s}^{\prime} u(k(Y))$ is purely transcendental over $L_{s} u(k(Y))$ and $\sigma_{s}^{\prime} \in G_{F \mid L_{s} u(k(Y))}$.

Corollary 3.3. For any $W \in \mathcal{I}_{G}$ the complex $0 \rightarrow W^{G} \rightarrow W^{G_{F \mid k(Y)}} \rightarrow W^{G_{F \mid k\left(Y^{2}\right)}} \rightarrow \ldots$ is exact. In particular, $0 \rightarrow C H^{q}(X)_{\mathbb{Q}} \rightarrow C H^{q}\left(X_{k(Y)}\right)_{\mathbb{Q}} \rightarrow C H^{q}\left(X_{k\left(Y^{2}\right)}\right)_{\mathbb{Q}} \rightarrow \ldots$ is exact.

Proof. The complex $\operatorname{Hom}_{G}\left(C_{k(Y \bullet)}, W\right)$ is exact, since, by Lemma 3.2, $\left.C_{k(Y} \bullet\right)$ is a projective resolution of 0 in $\mathcal{I}_{G}$.

Corollary 3.4. Sending the function field $L$ of a k-variety to $\mathbb{Q}[\{L \stackrel{/ k}{\hookrightarrow} F\}] \in \mathcal{S} m_{G}$, resp. to $C_{L} \in \mathcal{I}_{G}$, defines a sheaf on $\mathfrak{D} m_{k}$ with values in $\mathcal{S} m_{G}^{\mathrm{op}}$, resp. in $\mathcal{I}_{G}^{\mathrm{op}}$. (Since $\mathcal{I}$ is right exact.)

Denote by $\check{H}^{*}(X,-)$ the Čech cohomology, i.e. $\lim \check{H}_{\mathfrak{U}}^{*}(X,-)$ for $\mathfrak{U}$ running over the covers of $X$.

Corollary 3.5 ([Mi] Ch.III, Corollary 2.5). $\check{H}^{*}$ coincides with $H^{*}$ for any sheaf if and only if $\check{H}^{*}$ transforms any short exact sequence of sheaves to a long exact sequence of Čech cohomologies.

For any extension $K$ of $k$ in $F$ with $F$ of infinite transcendence degree over $K$ fix a transcendence basis $\left\{x_{1}, x_{2}, x_{3}, \ldots\right\}=\left\{x_{1}^{(\bar{K})}, x_{2}^{(\bar{K})}, x_{3}^{(\bar{K})}, \ldots\right\}$ of $F$ over $K$. For each $m \geq 0$ set 
$F_{m}=\overline{K\left(x_{2^{m}}, x_{2^{m} \cdot 3}, x_{2^{m} \cdot 5}, \ldots\right)}$, and for each $j \geq 0$ fix a self-embedding $\sigma_{j}: F \stackrel{\sqrt{K}}{\hookrightarrow} F$ such that $\left.\sigma_{j}\right|_{F_{s}}=i d$ if $j>s$ and $\left.\sigma_{j}\right|_{F_{s}}=\left.\sigma_{s}\right|_{F_{s}}: F_{s} \stackrel{\sim}{\longrightarrow} F_{s+1}$ if $j \leq s$. For any extension $L$ of $K$ we fix $L_{0} \subseteq F_{0}$ isomorphic to $L$ over $K$ and set $L_{s}:=\sigma_{0}^{s}\left(L_{0}\right)$.

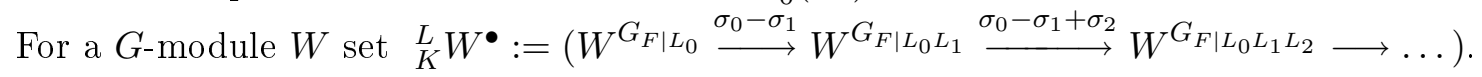

Proposition 3.6. One has $H_{\mathcal{S} m_{G}}^{q}\left(G_{F \mid K}, W\right)=H^{q}\left({ }_{K}^{F} W^{\bullet}\right)$ for any smooth representation $W$ of $G$ and any algebraically closed extension $K \supseteq k$ in $F$.

Proof. According to Corollary 3.5, it suffices to check that the functor $W \longmapsto H^{q}\left(\underset{K}{F} W^{\bullet}\right)$ coincides with the Čech cohomology $\lim H^{q}\left(\mathcal{F}(U) \rightarrow \mathcal{F}\left(U \times_{k} U\right) \rightarrow \mathcal{F}\left(U \times_{k} U \times_{k} U\right) \rightarrow \ldots\right)$ for the sheaf $\mathcal{F}$ on $\mathfrak{D} m_{k}$ corresponding to $W$, and transforms any short exact sequence of representations to a long exact sequence of spaces. By Lemma 5.7 of [R1], the functor $W \longmapsto{ }_{K}^{F} W^{\bullet}$ from $\mathcal{S} m_{G}$ to the category of complexes of $\mathbb{Q}$-vector spaces is exact, so the latter property follows.

If $K$ is algebraically closed in $L$ and $L$ is of finite type over $K$ then ${ }_{K}^{L} W^{\bullet}$ is isomorphic to the Čech complex $\operatorname{Hom}_{G}\left(\check{C}_{\bullet}^{L \mid K}, W\right)$ of the cover $\operatorname{Spec}(L) \longrightarrow \operatorname{Spec}(K)$ with coefficients in $\mathcal{F}_{W}$. Therefore, under these assumptions, $W \longmapsto H^{q}\left({ }_{K}^{L} W^{\bullet}\right)$ is the Čech cohomology of the cover $\operatorname{Spec}(L) \longrightarrow$ $\operatorname{Spec}(K)$. As $\frac{F}{K} W^{\bullet}=\lim _{L \longrightarrow} \frac{L}{K} W^{\bullet}$, we see that $H^{q}\left(\frac{F}{K} W^{\bullet}\right)=\lim _{L} H^{q}\left(\frac{L}{K} W^{\bullet}\right)$, where $L$ runs over the set of subfields in $F$ of finite type over $\bar{K}$. And finally, the functor $W \longmapsto H^{q}\left(\frac{F}{K} W^{\bullet}\right)$ coincides with the Čech cohomology of $\operatorname{Spec}(\bar{K})$.

EXAmples. 1. Let $W$ be a smooth $G$-module with trivial restriction to $G_{F \mid \bar{k}}$. Then $\mathcal{F}_{W}=$ $\left.\pi^{-1} \mathcal{F}_{W}\right|_{\operatorname{Spec}(k)_{\text {ét }}}$, where $\mathfrak{D} m_{k} \stackrel{\pi}{\longrightarrow} \operatorname{Spec}(k)_{\text {ét }}$ sends a smooth $k$-variety $U$ to the maximal $k$-scheme $U^{\prime}$ étale over $\operatorname{Spec}(k)$ in the Stein decomposition $U \longrightarrow U^{\prime} \longrightarrow \operatorname{Spec}(k)$.

For any connected cover $U \longrightarrow \operatorname{Spec}(\bar{k})$ the Čech complex with coefficients in $\mathcal{F}_{W}$ is just $W \stackrel{0}{\longrightarrow}$ $W \stackrel{i d}{\longrightarrow} W \stackrel{0}{\longrightarrow} W \stackrel{i d}{\longrightarrow} \ldots$, so $\check{H}_{\mathfrak{D} m_{\bar{k}}}^{>0}\left(\mathcal{F}_{W}\right)=0$.

Then the Hochschild-Serre spectral sequence $E_{2}^{p, q}=H^{p}\left(G_{\bar{k} \mid k}, H^{q}\left(G_{F \mid \bar{k}}, W\right)\right)$ is degenerate: $E_{2}^{p,>0}=0$, and thus, $\check{H}_{\mathfrak{D} m_{k}}^{*}\left(\mathcal{F}_{W}\right)=E_{2}^{*, 0}=H_{\text {êt }}^{*}\left(\operatorname{Spec}(k),\left.\mathcal{F}_{W}\right|_{\operatorname{Spec}(k)_{\text {ét }}}\right)$.

2. The sheaf $L \mapsto \mathbb{Q}[\{L \stackrel{/ k}{\hookrightarrow} F\}]$ (see Corollary 3.4) with values in $\mathcal{S} m_{G}^{\text {op }}$ is acyclic.

3. Let $A$ be an abelian variety over $k$. Then for any smooth variety $U$ over $k$ and its smooth compactification $\bar{U}$ the Čech complex $B \stackrel{d_{1}}{\longrightarrow} B^{2} \stackrel{d_{2}}{\longrightarrow} B^{3} \stackrel{d_{3}}{\longrightarrow} \ldots$ of $A(F) / A(k)$, where $B^{q}=$ $\operatorname{Hom}\left(\operatorname{Alb}\left(\bar{U}^{q}\right), A\right)$, is acyclic, since

$$
d_{q}:\left(b_{1}, \ldots, b_{q}\right) \mapsto \begin{cases}\left(0, b_{1}+b_{2}, 0, b_{3}+b_{4}, 0, \ldots, 0, b_{q-1}+b_{q}, 0\right) & \text { if } q \text { is even } \\ \left(-b_{1}, b_{1},-b_{3}, b_{3}, \ldots,-b_{q}, b_{q}\right) & \text { if } q \text { is odd }\end{cases}
$$

More generally, fix $n \geq 0$ and set $C^{q}=H^{n}\left(\bar{U}^{q}\right) / N^{1}$. The complex $C^{\bullet}$ is the direct sum of the subcomplexes $C_{m_{1}, \ldots, m_{s}}^{\bullet}$ for all collections $\left(m_{1}, \ldots, m_{s}\right)$ such that $m_{1}, \ldots, m_{s} \geq 1$ and $m_{1}+\cdots+m_{s}=n$,

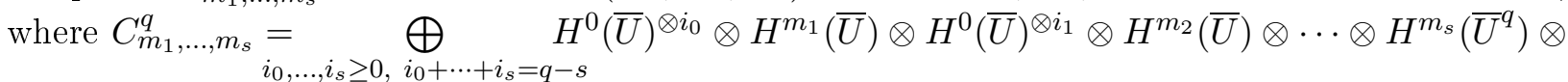
$H^{0}(\bar{U})^{\otimes i_{s}} / N^{1} \cong\left(C_{m_{1}, \ldots, m_{s}}^{s}\right)^{\left(\begin{array}{l}q \\ s\end{array}\right)}$. Clearly, all $C_{m_{1}, \ldots, m_{s}}^{\bullet}$ are acyclic.

3.1. 'Geometric' representations and the acyclicity. Any field extension $K$ of $k$ is a filtered union of finitely generated smooth $k$-subalgebras. Then the construction of $\$ 1$, preceding Lemma 1.1. associates to any presheaf $\mathcal{F}$ on $\mathfrak{D} m_{k}$ the smooth $\operatorname{Aut}(K \mid k)$-set $\mathcal{F}(K)$.

Lemma 3.7. One has $\mathcal{F}(F)^{G_{F \mid F^{\prime}}}=\mathcal{F}\left(F^{\prime}\right)$ for any $F^{\prime}=\overline{F^{\prime}} \subseteq F$ with $\operatorname{tr} \cdot \operatorname{deg}\left(F^{\prime} \mid k\right)=\infty$. 
(As it shows the example of the presheaf $\mathcal{F}: U \mapsto \Gamma\left(\bar{U}, \bigotimes_{\mathcal{O}}^{2} \Omega_{\bar{U} / k}^{1}\right)$, one cannot drop the condition that $F^{\prime}$ is algebraically closed. Here $\bar{U}$ is a smooth compactification of $U$.)

Proof. Fix an isomorphism $\alpha: F \stackrel{\sim}{\longrightarrow} F^{\prime}$ over $k$. Then $\alpha^{*}: \lim _{U \longrightarrow} \mathcal{F}(U) \stackrel{\sim}{\longrightarrow} \lim _{V \longrightarrow} \mathcal{F}(V)$, where $\mathcal{O}(U) \subset F$ and $\mathcal{O}(V) \subset F^{\prime}$ are smooth. For any $U$ there is $\sigma \in G$ such that $\left.\sigma\right|_{\mathcal{O}(U)}=\left.\alpha\right|_{\mathcal{O}(U)}$, so $\lim _{V \longrightarrow} \mathcal{F}(V)=\lim _{(U, \sigma)} \sigma \mathcal{F}(U)=\mathcal{F}(F)^{G_{F \mid F^{\prime}}}$

Note that the category of representations of type $\mathcal{F}(F)$ for $\mathcal{F}$ taking values in commutative groups is tensor. More generally, given a presheaf of commutative rings $\mathcal{A}$, the representations $\mathcal{F}(F)$ for the $\mathcal{A}$-modules $\mathcal{F}$ are $\mathcal{A}(F)$-modules and they form a tensor category with respect to the operation $\otimes_{\mathcal{A}(F)}$.

Assume that a presheaf $\mathcal{F}$ on $\mathfrak{D} m_{k}$ is endowed with transformations $i_{X, Y}^{x}: \mathcal{F}\left(X \times_{k} Y\right) \longrightarrow \mathcal{F}(Y)$ for any smooth $X, Y$ and any $x \in X(k)$ such that $i_{X, Y}^{x} \circ{ }_{X} \operatorname{pr}_{Y}^{*}=i d_{\mathcal{F}(Y)}$ and $i_{X, Y \times_{k} Z}^{x}{ }_{Z} \operatorname{pr}_{X \times_{k} Y}^{*}=$ $Z \operatorname{pr}_{Y}^{*} \circ i_{X, Y}^{x}$, where ${ }_{X} \operatorname{pr}_{Y}: X \times_{k} Y \longrightarrow Y$ is the projection.

Corollary 3.8. $H^{>0}(G, W)=0$ for any $W \in \mathcal{I}_{G} ; H^{1}(G, \mathcal{F}(F))=\{*\}$ for any group-valued $\mathcal{F}$; and $H^{>0}(G, \mathcal{F}(F))=0$ for any $\mathcal{F}$ with values in commutative groups.

Proof. By Proposition 3.6, $\check{H}^{*}(G,-)=H^{*}(G,-)$. By Corollary 3.3, $\check{H}^{*}(G, W)=W^{G}$.

It suffices to show that the complex $0 \rightarrow \mathcal{F}(k) \stackrel{d_{0}}{\longrightarrow} \mathcal{F}(X) \stackrel{d_{1}}{\longrightarrow} \mathcal{F}\left(X^{2}\right) \stackrel{d_{2}}{\longrightarrow} \mathcal{F}\left(X^{3}\right) \stackrel{d_{3}}{\longrightarrow} \ldots$ is exact for any smooth $k$-variety $X$, where $d_{q}=\sum_{i=0}^{q}(-1)^{i}\left(\operatorname{pr}_{q}^{i}\right)^{*}$ and $\operatorname{pr}_{q}^{i}: X^{q+1} \longrightarrow X^{q}$ omits the $i$-th multiple. Fix some $u \in X(k)$ and denote by $u_{q}$ the closed embedding $X^{q} \stackrel{u \times}{\hookrightarrow} X^{q+1}$. Then $\operatorname{pr}_{q}^{i} \circ u_{q}=u_{q-1} \circ \operatorname{pr}_{q-1}^{i-1}$ for any $1 \leq i \leq q$ and $\operatorname{pr}_{q}^{0} \circ u_{q}=i d_{X^{q}}$, so $u_{q}^{*} \circ d_{q}+d_{q-1} \circ u_{q-1}^{*}=i d_{\mathcal{F}\left(X^{q}\right)}$, where $u_{q}^{*}:=i_{X, X^{q}}^{u}$, i.e. $u_{\bullet}^{*}$ defines a contracting homotopy.

Examples of $\mathcal{F}(F)$ are $\bigotimes_{F}^{\bullet} \Omega_{F \mid k_{0}}^{1}, \bigotimes_{k_{0}}^{\bullet} \Omega_{F \mid k_{0}, \mathrm{cl}}^{1}, \bigotimes_{k_{0}}^{\bullet} \Omega_{F \mid k_{0} \text {,ex }}^{1}$ for any $k_{0} \subseteq k$ and $A(F)$ for any group $k$-variety $A$, or $A(F) / A(k)$ if $A$ is commutative. More examples are in the following

Corollary 3.9. Let $E$ be a field, endowed with a smooth $G$-action, which is presentable as $\mathcal{G}(F)$ for a presheaf of commutative rings $\mathcal{G}$ on $\mathfrak{D} m_{k}$ admitting transformations $i_{X, Y}^{x}$ as above.

(1) Let $B$ be such a family of fields of finite type over $k$ that any extension of $k$ of finite type can be embedded (over $k$ ) into an element of $B$.

Then the collection $\{E[\{L \stackrel{/ k}{\hookrightarrow} F\}]\}_{L \in B}$ is a system of acyclic generators of the category $\mathcal{S} m_{G}(E)$ (of smooth E-semilinear representations of $G$ ).

(2) The cohomological dimension of the category $\mathcal{S m}_{G}(E)$ is infinite.

Proof.

(1) For any field extension $L \mid k$ of finite type consider the functor $\mathcal{F}(X)=Z^{q}\left(X_{L}\right) \otimes \mathcal{G}(X)$, where $q=\operatorname{tr} \cdot \operatorname{deg}(L \mid k)$. Then $E[\{L \stackrel{/ k}{\hookrightarrow} F\}]=\mathcal{F}(F)$. Define the transformations $i_{X, Y}^{x}$ : $Z^{q}\left(X \times_{k} Y_{L}\right) \otimes \mathcal{G}\left(X \times_{k} Y\right) \longrightarrow Z^{q}\left(Y_{L}\right) \otimes \mathcal{G}(Y)$ by $\alpha \otimes g \mapsto \alpha \cap(\{x\} \times Y) \otimes i_{X, Y}^{x} g$ if the intersection is transversal, and $\alpha \otimes g \mapsto 0$ otherwise, for any irreducible $\alpha \subset X \times_{k} Y_{L}$.

(2) For any $q \geq 1$ and any object $W \in \mathcal{S} m_{G}(E)$ such that $H^{q}(G, W) \neq 0$ there is a surjection $\alpha: U \longrightarrow W$, where $U$ is a direct sum of acyclic generators of $\mathcal{S} m_{G}(E)$, cf. (11). Then there is an embedding $H^{q}(G, W) \hookrightarrow H^{q+1}(G$, $\operatorname{ker} \alpha)$, i.e., $H^{q+1}\left(G, W^{\prime}\right) \neq 0$ for $W^{\prime}=\operatorname{ker} \alpha$.

EXAmples. 1. $E=F=\mathcal{G}_{1}(F)$ with $\mathcal{G}_{1}(X)=\mathcal{O}(X)$ and $i_{X, Y}^{x}$ being the restriction to $\{x\} \times Y$. 
2. $\mathbb{Q} \subseteq E=\mathcal{G}_{2}(F) \subseteq k$ with $\mathcal{G}_{2}: X \mapsto E^{\pi_{0}(X)}$ being the sub-presheaf of $E$-valued locally constant functions in $\mathcal{G}_{1}$ and $i_{X, Y}^{x}$ being the restriction of the corresponding transformation on $\mathcal{G}_{1}$.

Conjecture 3.10. $\operatorname{Ext}_{\mathcal{S} m_{G}}^{*}\left(W_{1}, W_{2}\right)=\operatorname{Ext}_{\mathcal{I}_{G}}^{*}\left(W_{1}, W_{2}\right)$ for any $W_{1}, W_{2} \in \mathcal{I}_{G}$.

This is known for $* \leq 1$ (since $\mathcal{I}_{G}$ is a Serre subcategory of $\mathcal{S} m_{G}$ ) and for trivial $W_{1}$ (by Corollary 3.8).

3.2. $\mathbb{A}^{1}$-invariance of some presheaves. Let $\mathcal{V}_{k}$ be a category of $k$-varieties, containing all smooth varieties. Let $\mathcal{L}$ be a category, where all self-embeddings are isomorphisms (e.g., an abelian category such that for any object the multiplicities of its irreducible subquotients are finite 1 ).

An $\mathcal{L}$-valued presheaf $\mathcal{F}$ on $\mathcal{V}_{k}$ is $\mathbb{A}^{1}$-invariant, if $\mathcal{F}(U)=\mathcal{F}\left(U \times \mathbb{A}^{1}\right)$ for any $U \in \mathcal{V}_{k}$.

Consider any pretopology on $\mathcal{V}_{k}$ such that $\mathbb{A}_{k}^{1} \longrightarrow \operatorname{Spec}(k)$ is a covering and one of the following two conditions holds:

- $k$ contains all 2-primary roots of unity and there are no non-trivial divisible involutions in the group $\operatorname{Aut}(C)$ for any $C \in \mathcal{L}$ (e.g., if $\mathcal{L}$ is the category of finitely generated admissible 2 representations over a number field of a totally disconnected topological group);

- $\left(\mathbb{A}_{k}^{1} \backslash\{0\}\right) \amalg\left(\mathbb{A}_{k}^{1} \backslash\{1\}\right) \stackrel{i d \sqcup i d}{\longrightarrow} \mathbb{A}_{k}^{1}$ and $\mathbb{G}_{m, k} \longrightarrow \mathbb{G}_{m, k}, x \mapsto x^{2}$, are coverings (in particular, $\mathcal{F}\left(\mathbb{A}_{X}^{1}\right) \longrightarrow \mathcal{F}\left(\mathbb{G}_{m, X}\right)$ is injective for any $X \in \mathcal{V}_{k}$ and any sheaf on $\left.\mathcal{V}_{k}\right)$.

Proposition 3.11. Let $\mathcal{F}$ be a sheaf on $\mathcal{V}_{k}$ with values in $\mathcal{L}$. Then $\mathcal{F}$ is $\mathbb{A}^{1}$-invariant.

Proof. Let $U \in \mathcal{V}_{k}$ and $\sigma$ be the involution of $\mathcal{F}\left(U \times \mathbb{A}^{1} \times \mathbb{A}^{1}\right)$ induced by the interchanging of the two multiples $\mathbb{A}^{1}$, so $\mathrm{pr}_{1}^{*}=\sigma \circ \mathrm{pr}_{2}^{*}$, where $\mathrm{pr}_{1}, \mathrm{pr}_{2}: U \times \mathbb{A}^{1} \times \mathbb{A}^{1} \rightrightarrows U \times \mathbb{A}^{1}$ are the projections.

As $U \times \mathbb{A}^{1} \longrightarrow U$ is a covering, we get that $\mathcal{F}(U)$ is the equalizer of the injections

$$
\mathrm{pr}_{1}^{*}, \mathrm{pr}_{2}^{*}: \mathcal{F}\left(U \times \mathbb{A}^{1}\right) \rightrightarrows \mathcal{F}\left(U \times \mathbb{A}^{1} \times \mathbb{A}^{1}\right) .
$$

If $k$ contains all 2-primary roots of unity then $\sigma$ is a divisible involution in the group $\operatorname{Aut}_{\mathcal{L}}(\mathcal{F}(U \times$ $\left.\left.\mathbb{A}^{1} \times \mathbb{A}^{1}\right)\right)$.

If the Zariski covering $\mathbb{G}_{m, k} \amalg \mathbb{G}_{m, k} \longrightarrow \mathbb{A}_{k}^{1}$ is a covering in $\mathcal{V}_{k}$ then $\mathcal{F}\left(U \times \mathbb{A}^{1} \times \mathbb{A}^{1}\right) \longrightarrow$ $\mathcal{F}\left(U \times\left(\left(\mathbb{A}^{1} \times \mathbb{A}^{1}\right) \backslash \Delta_{\mathbb{A}^{1}}\right)\right)$ is injective, since $\left(\mathbb{A}^{1} \times \mathbb{A}^{1}\right) \backslash \Delta_{\mathbb{A}^{1}} \stackrel{\sim}{\longrightarrow} \mathbb{A}^{1} \times \mathbb{G}_{m},(x, y) \mapsto(x, x-y)$.

The morphism $U \times\left(\left(\mathbb{A}^{1} \times \mathbb{A}^{1}\right) \backslash \Delta_{\mathbb{A}^{1}}\right) \longrightarrow U \times\left(\left(\mathbb{A}^{1} \times \mathbb{A}^{1}\right) \backslash \Delta_{\mathbb{A}^{1}}\right) / \mathfrak{S}_{2}$ in the category $\mathcal{V}_{k}$ induces a $\mathfrak{S}_{2}$-invariant morphism $\mathcal{F}\left(U \times\left(\left(\mathbb{A}^{1} \times \mathbb{A}^{1}\right) \backslash \Delta_{\mathbb{A}^{1}}\right) / \mathfrak{S}_{2}\right) \longrightarrow \mathcal{F}\left(U \times\left(\left(\mathbb{A}^{1} \times \mathbb{A}^{1}\right) \backslash \Delta_{\mathbb{A}^{1}}\right)\right)$ in $\mathcal{L}$, which is injective if $\mathbb{G}_{m, k} \longrightarrow \mathbb{G}_{m, k}, x \mapsto x^{2}$, is a covering. As $\left(\left(\mathbb{A}^{1} \times \mathbb{A}^{1}\right) \backslash \Delta_{\mathbb{A}^{1}}\right) / \mathfrak{S}_{2} \stackrel{\sim}{\longrightarrow}\left(\mathbb{A}^{1} \times \mathbb{A}^{1}\right) \backslash \Delta_{\mathbb{A}^{1}}$, $(x, y) \mapsto\left(x+y, x+y+(x-y)^{2}\right)$, the involution $\sigma$ acts trivially on $\mathcal{F}\left(U \times\left(\left(\mathbb{A}^{1} \times \mathbb{A}^{1}\right) \backslash \Delta_{\mathbb{A}^{1}}\right)\right)$ by the assumptions on $\mathcal{L}$.

In any case, one has $\mathrm{pr}_{1}^{*}=\sigma \circ \operatorname{pr}_{2}^{*}=\operatorname{pr}_{2}^{*}$, and thus, $\mathcal{F}(U) \longrightarrow \mathcal{F}\left(U \times \mathbb{A}^{1}\right)$ is an isomorphism.

Example. If a morphism $p: U \longrightarrow Y$ in $\mathcal{V}_{k}$ admits a section $s: Y \longrightarrow U$ (e.g. for $U=Y \times_{k} Z$, and $p$ being the projection to $Y$, where $Z$ has a rational $k$-point) then the sequence $\mathcal{G}(Y) \stackrel{p^{*}}{\longrightarrow} \mathcal{G}(U) \rightrightarrows$ $\mathcal{G}\left(U \times_{Y} U\right)$ is exact for any presheaf $\mathcal{G}: p^{*}$ is injective, since the identity composition $Y \longrightarrow U \longrightarrow Y$ induces identity composition $\mathcal{G}(Y) \longrightarrow \mathcal{G}(U) \longrightarrow \mathcal{G}(Y) ; p^{*}$ is an equalizer of $\mathcal{G}(U) \rightrightarrows \mathcal{G}\left(U \times{ }_{Y} U\right)$, since the compositions $U \stackrel{(i d, s p)}{\longrightarrow} U \times_{Y} U \rightrightarrows U$ induce the identical and $(s p)^{*}=p^{*} s^{*}$ compositions $\mathcal{G}(U) \rightrightarrows \mathcal{G}\left(U \times_{Y} U\right) \longrightarrow \mathcal{G}(U)$, i.e. any element in the equalizer belongs to the image of $p^{*}$.

\footnotetext{
${ }^{1}$ The multiplicity of an irreducible object $X$ in $W$ is defined inductively: it is 0 if for any filtration $W \supseteq Y \supseteq Z$ the quotient $Y / Z$ is not isomorphic to $X$; it is $N>0$ if there is a filtration $W \supseteq Y \supseteq Z$ such that $Y / Z \cong X$ and the sum the multiplicities of $X$ in $W / Y$ and in $Z$ is $N-1$. By Jordan-Hölder theorem, the multiplicity is well-defined.

${ }^{2} \mathrm{~A}$ smooth representation of a totally disconnected topological group is called admissible if any open subgroup fixes a finite-dimensional subspace in it.
} 
Question. Any cohomology theory $H^{*}$ on $\mathcal{V}_{k}$ with coefficients in a field and any $q \geq 0$ define an $\mathbb{A}^{1}$-invariant presheaf $H^{q}$ on $\mathcal{V}_{k}$. We want it to be a sheaf in some topology. Can one simply define the covering morphisms as smooth morphisms $p: U \longrightarrow X$ such that the sequence $0 \longrightarrow$ $H^{*}(Y) \longrightarrow H^{*}\left(U^{\prime}\right) \longrightarrow H^{*}\left(U^{\prime} \times_{Y} U^{\prime}\right)$ is exact for any base change $U^{\prime} \longrightarrow Y$ of $p$. 3 In particular, no non-surjective morphism in $\mathcal{V}_{k}$ is covering, if one allows the closed embeddings as morphisms in $\mathcal{V}_{k}$. (If $Y$ is a point of $X$, which is not covered, then $U^{\prime}$ is empty, so the sequence $0 \longrightarrow H^{0}(Y) \longrightarrow$ $H^{0}\left(U^{\prime}\right) \longrightarrow H^{0}\left(U^{\prime} \times_{Y} U^{\prime}\right)$ is not exact.) It follows that this is the case for arbitrary morphisms admitting a section $U \longrightarrow X \mathbb{A}$ Clearly, the Zariski coverings are not coverings for such topology ( $\mathbb{P}^{n}$ is a union of $n+1$ copies of $\mathbb{A}^{n}$, but $H^{>0}\left(\mathbb{P}^{n}\right) \longrightarrow H^{>0}\left(\mathbb{A}^{n}\right)^{n+1}=0$ is not injective); the fibration $\mathbb{A}^{n+1} \backslash\{0\} \longrightarrow \mathbb{P}^{n}$ is not covering, since $H^{2 n}\left(\mathbb{P}^{n}\right) \longrightarrow H^{2 n}\left(\mathbb{A}^{n+1} \backslash\{0\}\right)=0$ is not injective.

\section{The site $\mathfrak{H}$ (the Hartogs topology)}

Let $v: F^{\times} / k^{\times} \longrightarrow \mathbb{Q}^{r}$ be a discrete valuation, i.e. $F$ is algebraic over the subfield generated by an isomorphic lifting of the residue field of $v$ and by an isomorphic lifting of the valuation group $\mathbb{Q}^{r}$. Let $G_{v}$ be the decomposition subgroup of $v$ in $G$, i.e., the stabilizer of the valuation $k$-algebra $\mathcal{O}_{v} \subset F$. For any $W \in \mathcal{S} m_{G}$ set $W_{v}:=\sum_{\sigma \in G_{v}} W^{G_{F \mid \sigma\left(F^{\prime}\right)}} \subseteq W$. The additive functor $\mathcal{S} m_{G} \longrightarrow \mathcal{S} m_{G_{v}}, W \mapsto W_{v}$, is fully faithful and preserves surjections and injections, cf. [R3, §4.1].

Let $\Gamma_{r}(W)$ be the intersection of $W_{v}$ over all discrete valuations $v: F^{\times} / k^{\times} \longrightarrow \mathbb{Q}^{r}$. Set $\Gamma:=\Gamma_{1}$, so $\Gamma_{r}: \mathcal{S} m_{G} \longrightarrow \mathcal{S} m_{G}$ are additive functors.

ExAmples. $\mathbb{Q}[\{L \stackrel{/ k}{\hookrightarrow} F\}]_{v}=\mathbb{Q}\left[\left\{L \stackrel{/ k}{\hookrightarrow} \mathcal{O}_{v}\right\}\right] ; F[\{L \stackrel{/ k}{\hookrightarrow} F\}]_{v}=\mathcal{O}_{v}\left[\left\{L \stackrel{/ k}{\hookrightarrow} \mathcal{O}_{v}\right\}\right]$.

Proposition 4.1 ([R] $)$. $\Gamma(W)=W_{v}=W$ for any $W \in \mathcal{I}_{G}$.

Lemma $4.2([\mathrm{R} 3])$. One has $\left(W_{1} \otimes W_{2}\right)_{v} \subseteq\left(W_{1}\right)_{v} \otimes\left(W_{2}\right)_{v}$ and $\Gamma\left(W_{1} \otimes W_{2}\right) \subseteq \Gamma\left(W_{1}\right) \otimes \Gamma\left(W_{2}\right)$ for any $W_{1}, W_{2} \in \mathcal{S} m_{G}$. However, $(W \otimes W)_{v} \neq W_{v} \otimes W_{v}$ if $W=\mathbb{Q}[F \backslash k]$.

If either $W_{1}$ is a quotient of $A(F)$ for a commutative algebraic k-group $A$, or $W_{1} \in \mathcal{I}_{G}$ then $\left(W_{1} \otimes W_{2}\right)_{v}=\left(W_{1}\right)_{v} \otimes\left(W_{2}\right)_{v}$ for any $W_{2} \in \mathcal{S} m_{G}$.

Remarks. 1. By Proposition 4.1 and Lemma 4.2, $\Gamma(W \otimes F)=W \otimes k$ for any $W \in \mathcal{I}_{G}$, so $\Gamma(V) \otimes F \longrightarrow V$ for any semilinear quotient $V$ of $W \otimes F$.

2. If $W$ is an $F$-vector space then the $F$-vector space structure $F \otimes W \longrightarrow W$ on $W$ induces an $\mathcal{O}_{v}$-module structure $(F \otimes W)_{v}=\mathcal{O}_{v} \otimes W_{v} \longrightarrow W_{v}$ on $W_{v}$. Clearly, $F \otimes \mathcal{O}_{v} W_{v} \longrightarrow W$ is injective, but not surjective, as shows the example of $W=F[\{L \stackrel{/ k}{\hookrightarrow} F\}]$.

3. Clearly, $\Gamma_{r}$ preserves the injections, but not the surjections. Namely, let $W:=\bigotimes_{k}^{N} F \longrightarrow$ $\Omega_{F \mid k}^{N-1}$ be given by $a_{1} \otimes \cdots \otimes a_{N} \mapsto a_{1} d a_{2} \wedge \cdots \wedge d a_{N}$. By Lemma 4.2, $W_{v}=\bigotimes_{k}^{N} \mathcal{O}_{v}$, so $\left(\bigotimes_{F}^{N-1} \Omega_{F \mid k_{0}}^{1}\right)_{v}=\bigotimes_{F}^{N-1} \Omega_{\mathcal{O}_{v} \mid k_{0}}^{1}$ for any $k_{0} \subseteq k$; and $\Gamma\left(\bigotimes_{k}^{N} F\right)=k$, but $\Gamma_{r}\left(\Omega_{F \mid k}^{\bullet}\right)=\Omega_{F \mid k \text {,reg }}^{\bullet}$ for any $r \geq 1$.

For an integral normal $k$-variety $X$ with $k(X) \subset F$ let $\mathfrak{V}(X)$ be the set of all discrete valuations of $F$ of rank one trivial on $k$ such that their restrictions to $k(X)$ are either trivial, or correspond to divisors on $X$. Set $\mathcal{W}(X):=W^{G_{F \mid k(X)}} \cap \bigcap_{v \in \mathfrak{V}(X)} W_{v} \subseteq W$.

REMARK. $W^{G_{F \mid k(X)}} \cap W_{v}$ depends only on the restriction of $v$ to $k(X)$, since the set of $G_{F \mid k(X)^{-}}$ orbits $G_{F \mid k(X)} \backslash G / G_{v}$ of the valuations of $F$ coincides with the set of valuations of $k(X)$ of rank $\leq r$. E.g., if the restriction of $v$ to $k(X)$ is trivial then $W^{G_{F \mid k(X)}} \subseteq W_{v}$.

\footnotetext{
${ }^{3}$ It is not clear, whether this is compatible with compositions.

${ }^{4}$ and clearly this type of coverings is stable under base changes and compositions
} 
Consider the following site $\mathfrak{H}$. Objects of $\mathfrak{H}$ are the smooth varieties over $k$. Morphisms in $\mathfrak{H}$ are the locally dominant morphisms, transforming non-dominant divisors to divisors. Coverings are smooth morphisms surjective over the generic point of any divisor on the target.

Lemma 4.3. A choice of $k$-embeddings into $F$ of all generic points of all smooth $k$-varieties defines a sheaf $\mathcal{W}$ on $\mathfrak{H}$ for any $W \in \mathcal{S} m_{G}$.

Proof. Clearly, if a dominant morphism $f: U \longrightarrow X$ transforms divisors on $U$, non-dominant over $X$, to divisors on $X$ then $\mathfrak{V}(U) \subseteq \mathfrak{V}(X)$, so $\mathcal{W}(X) \subseteq \mathcal{W}(U)$.

If, moreover, the pull-back of any divisor on $X$ is a divisor on $U$ then $\mathfrak{V}(X)=\mathfrak{V}(U)$.

By Lemma 1.1, $X \mapsto \prod_{x \in X^{0}} W^{G_{F \mid k(x)}}$ is a sheaf on $\mathfrak{D} m_{k}$, so the sequence

$$
0 \longrightarrow \prod_{x \in X^{0}} W^{G_{F \mid k(x)}} \longrightarrow \prod_{x \in U^{0}} W^{G_{F \mid k(x)}} \longrightarrow \prod_{x \in\left(U \times_{X} U\right)^{0}} W^{G_{F \mid k(x)}}
$$

is exact. As $\mathfrak{V}(X)=\mathfrak{V}(U)=\mathfrak{V}\left(U \times_{X} U\right)$, the sheaf property for the covering $f$ amounts to the exactness of the above sequence restricted to $\prod_{x \in U^{0}} \bigcap_{v \in \mathfrak{V}(X)} W_{v}$.

Examples. $X \mapsto \Gamma\left(X, \Omega_{X \mid k}^{q}\right)$ and $X \mapsto \Gamma\left(\bar{X}, \Omega_{\bar{X} \mid k}^{q}\right)$ are sheaves on $\mathfrak{H}$ for any integer $q \geq 0$; $X \mapsto \Gamma\left(\bar{X}, \operatorname{Sym}^{2} \Omega \frac{q}{X \mid k}\right)$ is not a sheaf on $\mathfrak{H}$. Here $\bar{X}$ is a smooth compactification of $X$.

\section{Appendix A. Totally Disconnected groups And their REPRESENTATions}

Let $H$ be an arbitrary totally disconnected topological group, and $B$ a base of its open subgroups. By definition, this means that $B$ is a collection of subgroups of $H$ such that (i) it has the trivial intersection, (ii) the conjugate of each subgroup in $B$ by any element of $H$ contains a subgroup in $B$, (iii) each finite intersections of subgroups in $B$ contains a subgroup in $B$. Then a subgroup of $H$ is called open if it contains an element of $B$ (so $H$ is Hausdorff).

Let $E$ be a field of characteristic zero endowed with a smooth (e.g., trivial) $H$-action.

An example of $H$ is given by a permutation group of a set (with the stabilizers of finite subsets as a base of open subgroups). This example is typical in the sense that arbitrary $H$ is a permutation group of the disjoint union $\coprod_{U \in B} H / U$ for arbitrary collection $B$ of subgroups of $H$ with trivial intersection of their conjugates, e.g., for a base of open subgroups of any totally disconnected group $H$. If there is an open subgroup $U$ of $H$ containing no nontrivial normal subgroups of $H$ then $H$ is a permutation group of the set $H / U$.

In this appendix we make some general remarks on the category $\mathcal{S} m_{H}(E)$ of $E$-vector spaces endowed with a smooth semilinear $H$-action, generalizing some well-known facts about locally compact totally disconnected groups.

A.1. Smooth $H$-sets and sheaves. It is well-known (e.g., [SGA 4 I, Exposé IV, §2.4-2.5] or [Joh, $\S 8.1$, Example 8.15 (iii)]) that the smooth $H$-sets and their $H$-equivariant maps form a topos.

Let $\mathfrak{T}=\mathfrak{T}(H, B)$ be the category whose objects are the elements of some base $B$ of open subgroups of $H$ and $\operatorname{Hom}_{\mathfrak{T}}(U, V)=\left\{h \in H \mid h U h^{-1} \supseteq V\right\} / U$. The composition is defined in the natural way: if $U \stackrel{\alpha}{\longrightarrow} V \stackrel{\beta}{\longrightarrow} W$, i.e., $\alpha U \alpha^{-1} \supseteq V$ and $\beta V \beta^{-1} \supseteq W$, then $\beta \alpha U(\beta \alpha)^{-1} \supseteq W(\beta v \alpha u=$ $\beta \alpha\left(\alpha^{-1} v \alpha\right) u \in \beta \alpha U$ for any $u \in U$ and $v \in V$, so the composition is well-defined). We endow $\mathfrak{T}$ with the maximal topology, i.e. we assume that any sieve is covering. Then the sheaves of sets, groups, etc. on $\mathfrak{T}$ are identified with the smooth $H$-sets, groups, etc.: $\mathcal{F} \mapsto \lim _{\overrightarrow{U \in B}} \mathcal{F}(U)$ (this is a smooth $H$-set, since its arbitrary element belongs to the image of some $\mathcal{F}(U)$ and the $U$-action on it is trivial by definition) and $W \mapsto\left(U \mapsto W^{U}\right)$. 
Examples. 1. If $H$ is discrete and $B=\{1\}$ then there is exactly one object $*$ in $\mathfrak{T}$ and $\operatorname{Hom}_{\mathfrak{T}}(*, *)=H$.

2. In notation of Introduction, the set $\operatorname{Hom}_{\mathfrak{T}(G, B)}\left(G_{F \mid L}, G_{F \mid K}\right)=\{h \in G \mid h(L) \subseteq K\} / G_{F \mid L}$ consists of all field embeddings $L \stackrel{/ k}{\hookrightarrow} K$ over $k$. (Here $B$ is the set of open subgroups in $G$ of type $G_{F \mid L}$, where $L \mid k$ is an extension of finite type.)

A.2. Basic structures on $\mathcal{S} m_{H}(E)$. There are direct sums, direct products and the inner $\mathcal{H}$ om functor in the categories $\mathcal{S}_{H}(E)$ and $\mathcal{I}_{G}$. They are the smooth parts of the corresponding functors on the category of $E$-vector spaces, and therefore, these functors on $\mathcal{I}_{G}$ coincide with the restrictions of the corresponding functors on $\mathcal{S} m_{G}(\mathbb{Q})$. Namely, the direct sums are direct sums in the category of abelian groups; the direct product of a family of objects is the smooth part of its set-theoretic direct product: $\prod_{\alpha}^{\mathcal{S} m_{H}} W_{\alpha}:=\bigcup_{U \in B} \prod_{\alpha} W_{\alpha}^{U}$, and $\mathcal{H o m}\left(W_{1}, W_{2}\right):=\varliminf_{U \in B} \operatorname{lom}_{E[U]}\left(W_{1}, W_{2}\right)$.

The usual tensor structure on the category of $E$-vector spaces induces a tensor structure on the category $\mathcal{S m}_{H}(E)$. The functor $\mathcal{H o m}(W,-)$ is right adjoint to the functor $-\otimes_{E} W$ :

$$
\begin{aligned}
\operatorname{Hom}_{\mathcal{S} m_{H}(E)}\left(W_{1} \otimes_{E} W, W_{2}\right)= & \operatorname{Hom}_{E[H]}\left(W_{1} \otimes_{E} W, W_{2}\right)=\operatorname{Hom}_{E[H]}\left(W_{1}, \operatorname{Hom}_{E}\left(W, W_{2}\right)\right)= \\
& =\operatorname{Hom}_{E[H]}\left(W_{1}, \mathcal{H o m}\left(W, W_{2}\right)\right)=\operatorname{Hom}_{\mathcal{S} m_{H}(E)}\left(W_{1}, \mathcal{H o m}\left(W, W_{2}\right)\right)
\end{aligned}
$$

for any $W_{1}, W_{2}, W \in \mathcal{S} m_{H}(E)$. The tensor product on $\mathcal{S} m_{G}(\mathbb{Q})$ does not preserve $\mathcal{I}_{G}$, but it has a unique modification $\otimes_{\mathcal{I}}$ with the above adjunction property, cf. [R1, §6.4]. The associativity of $\otimes_{\mathcal{I}}$, which is equivalent to the identity $\mathcal{H o m}\left(W_{1} \otimes W_{2},-\right)=\mathcal{H o m}\left(W_{1} \otimes_{\mathcal{I}} W_{2},-\right)$ on $\mathcal{I}_{G}$, is not yet known.

A.3. Generalities on Hecke algebras. Define $\mathbb{D}_{E}(H):=\lim _{U} E[H / U]$, where the projective system is formed with respect to the projection $E[H / V] \stackrel{r_{V U}}{\longrightarrow} E[H / U]$ and $H / V \longrightarrow H / U$, induced by the inclusions $V \subset U$ of open subgroups of $H$. In other words, $\mathbb{D}_{E}(H):=E \widehat{\otimes} \mathbb{D}_{\mathbb{Q}}(H)$.

Any element $\nu \in \mathbb{D}_{E}(H)$ can be considered as an $E$-valued 'oscillating' measure on $H$ (for which all open subgroups and their translates are measurable). In particular, for any $\sigma \in H$ and an open subgroup $U$ let the value $\nu(\sigma U)$ of the measure $\nu$ on the set $\sigma U$ be the $[\sigma U]$-coefficient in the image of $\nu$ in $E[H / U]$.

For each smooth semilinear $E$-representation $W$ of $H$ define a pairing $\mathbb{D}_{E}(H) \times W \longrightarrow W$ by $(\nu, w) \longmapsto \sum_{\sigma \in H / U} \nu(\sigma U) \cdot \sigma w$, where $U$ is an arbitrary open subgroup in the stabilizer of $w$, e.g., $U=\operatorname{Stab}_{w}$. Clearly, the result is independent of the choice of $U$. This determines a $\mathbb{D}_{E}(H)$-module structure on $W$. When $W=E[H / U]$, this pairing is compatible with the projections $r_{V U}$, so it gives rise to a pairing $\mathbb{D}_{E}(H) \times{\underset{\lim }{U}}_{U} E[H / U] \longrightarrow \longleftarrow_{U} E[H / U]=\mathbb{D}_{E}(H)$, and thus, to an associative multiplication $\mathbb{D}_{E}(H) \times \mathbb{D}_{E}(H) \stackrel{*}{\longrightarrow} \mathbb{D}_{E}(H)$, extending the convolution of the compactly supported measures. (The support of $\nu$ is the minimal closed subset $S$ in the semi-group $\widehat{H}:=\lim _{U} H / U$ such that $\nu(\sigma U)=0$ for any $\sigma U$ that does not meet $S$.)

Clearly, the construction of $\mathbb{D}_{E}(H)$ is functorial in the sense that the following data

- a totally disconnected group $H^{\prime}$,

- a field $E^{\prime}$ endowed with a smooth $H^{\prime}$-action,

- a continuous homomorphism $\varphi: H \longrightarrow H^{\prime}$,

- a smooth 1-cocycle $\theta: H \longrightarrow\left(E^{\prime}\right)^{\times}$, i.e., $\left.\theta\right|_{U}=1$ for some open subgroup $U \subset H$ and $\theta\left(h h^{\prime}\right)=\theta(h) \varphi(h)\left(\theta\left(h^{\prime}\right)\right)$,

- a field embedding $\lambda: E \longrightarrow E^{\prime}$, which is compatible with the $H$-action, 
induces a continuous homomorphism of algebras $\mathbb{D}_{E}(H) \longrightarrow \mathbb{D}_{E^{\prime}}\left(H^{\prime}\right), a[h] \mapsto \lambda a \cdot \theta(h)[\varphi h]$.

The Hecke algebra of a pair $(H, U)$, where $U$ is a compact subgroup of $H$, is the subalgebra $\mathcal{H}_{E}(H, U):=h_{U} * \mathbb{D}_{E}(H) * h_{U}$ in $\mathbb{D}_{E}(H)$ of $U$-biinvariant measures. Here $h_{U}$ is the Haar measure on $U$, defined by the system $\left(h_{U}\right)_{V}=[U: U \cap V]^{-1} \sum_{\sigma \in U / U \cap V}[\sigma V] \in \mathbb{Q}[H / V]$ for all open subgroups $V \subset H$. The element $h_{U}$ is the unity of the algebras $\mathcal{H}_{\mathbb{Q}}(H, U) \subseteq \mathcal{H}_{E}(H, U)$ and $h_{U} h_{U^{\prime}}=h_{U}$ for any closed subgroup $U^{\prime} \subseteq U$. For each smooth $E$-representation $W$ of $H$ the Hecke algebra $\mathcal{H}_{E}(H, U)$ acts on the space $W^{U}$, since $W^{U}=h_{U}(W)$.

This definition of the Hecke algebra is equivalent to the standard one in the case of locally compact $H$, open compact $U$ and a characteristic zero field $E$ endowed with the trivial $H$-action, and $\mathcal{H}_{E}(H, U)$ acts on the space $W^{U}$ in the usual way for any smooth $E$-representation $W$ of $H$, cf. [BZ].

If $H$ is the automorphism group of an algebraically closed extension of $k$ of finite transcendence degree $n$ then the Hecke algebras become the algebras of non-degenerate correspondences on some $n$-dimensional $k$-varieties, cf. Appendix B.

REMARKS. 1. There is the following evident modification to arbitrary totally disconnected groups of the standard semi-simplicity or irreducibility criteria, cf. [BZ, Proposition 2.10].

Let $T$ be a filtering family of compact subgroups of $H$, i.e. such that any open subgroup contains an element of $T$, e.g. the set of open subgroups of a given compact subgroup of $H$. Then a smooth $E$-representation $W$ of $H$ is irreducible, resp. semi-simple, if and only if the $\mathcal{H}_{E}(H, U)$-module $W^{U}$ is irreducible, resp. semi-simple, for each compact subgroup $U \in T$.

2. One uses the centres of the Hecke algebras to decompose the category of smooth representations. However, in the case of $H=G$ one can show (in a way similar to that of [R1, Appendix A, Theorem A.4]) that for any compact subgroup $K$ in $G$ the centre of the Hecke algebra $\mathcal{H}_{E}(K)$ of the pair $(G, K)$ coincides with $E \cdot h_{K}$, i.e., consists of scalars.

A.4. Pull-back functors. If $\varphi: H_{2} \longrightarrow H_{1}$ is a homomorphism with a dense image then the pull-back functor $\varphi^{-1}: \mathcal{S} m_{H_{1}} \longrightarrow H_{2}$-mod is fully faithful. (Proof. Let $W_{1}, W_{2} \in \mathcal{S} m_{H_{1}}, \alpha \in$ $\operatorname{Hom}_{H_{2}}\left(\varphi^{-1} W_{1}, \varphi^{-1} W_{2}\right), v \in W_{1}$ and $\sigma \in H_{1}$. Let $S$ be the common stabilizer of the elements $v$ and $\alpha(v)$. Choose some element $\sigma^{\prime} \in \varphi^{-1}(\sigma S) \subseteq H_{2}$. Then $\alpha(\sigma v)=\alpha\left(\sigma^{\prime} v\right)=\sigma^{\prime} \alpha(v)=\sigma \alpha(v)$.

If $\varphi$ is continuous then $\varphi^{-1}$ factors through $\varphi^{*}: \mathcal{S} m_{H_{1}} \longrightarrow \mathcal{S} m_{H_{2}}$.

If the homomorphism $\varphi$ is continuous and with dense image then the functor $\varphi^{*}$ admits a right adjoint $\varphi_{*}: W \mapsto \bigcup_{U} W^{U \times_{H_{1}} H_{2}}$, where $U$ runs over open subgroups of $H_{1}$. In particular, $\varphi^{*}$ preserves the irreducibility. (Proof. The $H_{1}$-action on $\varphi_{*} W$ is defined as follows. If $w \in W^{\varphi^{-1}(U)}$ and $\sigma \in H_{1}$ then $\sigma w:=\sigma^{\prime} w$, where $\sigma^{\prime} \in H_{2}$ and $\varphi\left(\sigma^{\prime}\right) \in \sigma U$, which is independent of $\sigma^{\prime}$.

Example. (A 'dense' locally compact 'subgroup' $\mathfrak{G}$ of $G$.) Let $\left\{x_{1}, x_{2}, \ldots\right\}$ be a transcendence base of $F \mid k$. Set $L_{m}:=k\left(x_{m}, x_{m+1}, \ldots\right) \subset F$. Then $L_{\bullet}=\left(L_{1} \supset L_{2} \supset L_{3} \supset \ldots\right)$ is a descending sequence of subfields in $F$. Set $\mathfrak{G}=\mathfrak{G}_{L_{\bullet}}:=\bigcup_{m \geq 1} G_{F \mid L_{m}}$. We take the set $\left\{G_{F \mid L L_{1}}\right\}$ of subgroups for all subfields $L$ in $F \mid k$ of finite type as a base of open subgroups.

Geometrically (in a sense, analogous to the dominant topology), this corresponds to an inverse system of infinite-dimensional irreducible $k$-varieties given by finite systems of equations. They are related by dominant morphisms affecting only finitely many coordinates.

Then

- $\mathfrak{G}$ is locally compact (since $F$ is algebraic over $L_{1}$ ), but is not unimodular;

- the inclusion $\mathfrak{G}$ into $G$ is continuous with dense image (since $\bigcap_{m \geq 1} \overline{L_{m}}=k$ ).

More details can be found in [R4]. 
The forgetful functor $\mathcal{S} m_{G} \longrightarrow \mathfrak{G}$-mod is fully faithful, preserves the irreducibility, factors through $r: \mathcal{S} m_{G} \longrightarrow \mathcal{S} m_{\mathfrak{G}}$, and $r$ admits a right adjoint: $W \mapsto \bigcup_{L} \bigcap_{m \geq 1} W^{G_{F \mid L L_{m}}}$, where $L$ runs over the set of all subfields of finite type in $F \mid k$.

A.5. Count of cyclic and irreducible objects of $\mathcal{S} m_{H}(E)$. Recall (e.g., [Mi, Ch. III, §1]) that a family $\left(A_{j}\right)_{j \in J}$ of objects in a category is generating if for any injection $\alpha: A^{\prime} \hookrightarrow A$ which is not an isomorphism there exist an index $j \in J$ and a morphism $A_{j} \longrightarrow A$ that does not factor through $\alpha$.

The objects $E[H / U]$, where $U \in B$, form a generating system of $\mathcal{S} m_{H}(E)$, i.e., any smooth cyclic $E$-semilinear representation of $H$ is a quotient of $E[H / U]$ for some $U \in B$.

One can modify the $H$-module structure on $E[H / U]$ by the rule $\sigma:[h] \mapsto f(\sigma, h) \cdot[\sigma h]$ for some function $f: H \times H / U \longrightarrow E^{\times}$. The associativity constraint gives the condition $f(\tau \sigma, h)=$ $\tau f(\sigma, h) \cdot f(\tau, \sigma h)$. In other words, $g(\sigma \tau, h)=g(\sigma, h) \cdot \sigma g\left(\tau, \sigma^{-1} h\right)$, where $g(\sigma, h)=\sigma f\left(\sigma^{-1}, h\right)$, i.e., $g \in Z^{1}\left(H, \operatorname{Maps}\left(H / U, E^{\times}\right)\right)$is a 1-cocycle on $H$. The smoothness constraint amounts to the condition that for any $h \in H / U$ there exists an open subgroup $V \subset H$ such that $f(\sigma, h)=1$ (equivalently, $g(\sigma, h)=1$ ) for any $\sigma \in V$. We denote by $E[H / U]^{(g)}$ the resulting object of $\mathcal{S} m_{H}(E)$.

Let $\delta: \operatorname{Maps}\left(H / U, E^{\times}\right) \longrightarrow Z^{1}\left(H, \operatorname{Maps}\left(H / U, E^{\times}\right)\right), \varphi \longmapsto\left[(\sigma, h) \mapsto \varphi(h)^{-1} \cdot \sigma \varphi\left(\sigma^{-1} h\right)\right]$, be the coboundary homomorphism. Any coboundary is smooth: $(\sigma, h) \mapsto \varphi(h)^{-1} \varphi(h)=1$ for any $\sigma \in \operatorname{Stab}_{\varphi(h)} \cap h U h^{-1}$. If two 1-cocycles are cohomological to each other then the corresponding objects of $\mathcal{S}_{H}(E)$ are isomorphic: $E[H / U]^{(g)} \stackrel{\sim}{\longrightarrow} E[H / U]^{(g \cdot \delta \varphi)},[h] \mapsto \varphi(h) \cdot[h]$.

Example. Suppose that $\varphi$ lifts to a 1-cocycle $H \longrightarrow E^{\times}$, and thus, corresponds to a onedimensional object $\mathcal{L}$ of $\mathcal{S} m_{H}(E)$. Then $(\delta \varphi)(\sigma, h)=\varphi(h)^{-1} \cdot \sigma \varphi\left(\sigma^{-1} h\right)=\varphi(h)^{-1} \varphi(\sigma)^{-1} \varphi(h)=$ $\varphi(h)^{-1}$, so $f(\sigma, h)=\sigma \varphi\left(\sigma^{-1}\right)^{-1}=\varphi(\sigma)$, and therefore, $E[H / U]^{(g \cdot \delta \varphi)} \cong E[H / U]^{(g)} \otimes_{E} \mathcal{L}$.

As the representations $E[H / U]^{(g)}$ and $E[H / U]^{(g \cdot \delta \varphi)}$ are isomorphic, this implies that for any irreducible $W \in \mathcal{S} m_{H}(E)$ and any one-dimensional $\mathcal{L} \in \mathcal{S} m_{H}(E)$ with $\mathcal{L}^{U} \neq 0$ the multiplicities of $W$ and of $W \otimes_{E} \mathcal{L}$ in $E[H / U]^{(g)}$ coincide.

It is likely, however, that these multiplicities are infinite, when $H$ is the automorphism group of a non-trivial algebraically closed extension of $k$ of finite transcendence degree, cf. [R1, Remark on p.217] (or [R4, p.1162]). More remarks on representations $E[G / U]$ are in Appendix B]

Proposition A.1. (1) There are at most $\max \left(|B|, \sup _{U \in B} 2^{\max (|H / U|,|E|)}\right)$ isomorphism classes of smooth cyclic E-semilinear representations of $H$.

(2) Suppose that $U$ is an open subgroup of $H$, and there are $>\sup _{U^{\prime} \in B} \max \left(\left|H / U^{\prime}\right|,|E|\right)$ isomorphism classes of irreducible objects of $\mathcal{S} m_{U}(E)$. Then the group $H$ admits at least as many isomorphism classes of smooth irreducible representations as $U$ does.

(3) Let $U$ be an open subgroup of $H$. Suppose that there are at least $\max \left(|B|, \sup _{U^{\prime} \in B} 2^{\max \left(\left|H / U^{\prime}\right|,|E|\right)}\right)$ isomorphism classes of irreducible objects of $\mathcal{S} m_{U}(E)$. Then the cardinality of the set of isomorphism classes of irreducible objects of $\mathcal{S} m_{H}(E)$ is equal to $\max \left(|B|, \sup _{U^{\prime} \in B} 2^{\max \left(\left|H / U^{\prime}\right|,|E|\right)}\right)$.

Proof. As $E[S]$ is dominated by $\bigcup_{N \geq 1} E^{N} \times S^{N},\left(a_{1}, \ldots, a_{N} ; s_{1}, \ldots, s_{N}\right) \mapsto \sum_{i=1}^{N} a_{i}\left[s_{i}\right]$, one has $|E[S]|=\max (|S|,|E|)$, if $E$ and $S$ are infinite. Then for any subgroup $U$ of $H$ there are $\leq 2^{\max (|H / U|,|E|)}$ quotients of the representation $E[H / U]$, which proves (1).

Let $U$ be an open subgroup of $H$, and $\varphi$ be a smooth cyclic E-representation of $U$. Let $W$ be any quotient of the cyclic representation $E[H] \otimes_{E[U]} \varphi$ of $H$. Then there are $\leq|W| \leq$ $\sup _{U^{\prime} \in B}\left|E\left[H / U^{\prime}\right]\right| \leq \sup _{U^{\prime} \in B} \max \left(\left|H / U^{\prime}\right|,|E|\right)$ isomorphism classes of cyclic subrepresentations of 
$U$ in $W$, one of which is $\varphi$, if $\varphi$ is irreducible. For each smooth irreducible representation $\varphi$ of $U$ choose an irreducible quotient $W_{\varphi}$ of the representation $E[H] \otimes_{E[U]} \varphi$.

We say that smooth irreducible representations of $U$ are equivalent (notation: $\varphi \sim \psi$ ) if $W_{\varphi} \cong$ $W_{\psi}$. As $\left|W_{\varphi}\right| \leq \sup _{U^{\prime} \in B} \max \left(\left|H / U^{\prime}\right|,|E|\right)$, the cardinalities of the equivalence classes do not exceed sup $\max \left(\left|H / U^{\prime}\right|,|E|\right)$. Therefore, $|\{\varphi\} / \sim|=|\{\varphi\}|$ under assumption of (2). $U^{\prime} \in B$

Under assumption of (3), the set of isomorphism classes of smooth irreducible representations of $U$ and the set of equivalence classes of smooth irreducible representations of $U$ have the same cardinality, and the lower bound $\geq \max \left(|B|, \sup _{U^{\prime} \in B} 2^{\max \left(\left|H / U^{\prime}\right|,|E|\right)}\right)$ of this cardinality coincides with the upper bound of the cardinality of the set of isomorphism classes of smooth irreducible representations of $H$ from (1), so the group $H$ admits precisely $\max \left(|B|, \sup _{U^{\prime} \in B} 2^{\max \left(\left|H / U^{\prime}\right|,|E|\right)}\right)$ isomorphism classes of smooth irreducible representations.

ExAmples. 1. Let $H$ be the automorphism group of a non-trivial algebraically closed field extension $F$ of $k$ of transcendence degree at most $|k|$, e.g., countable. Let $B$ be the set of stabilizers of finite subsets in $F$. Then $|B|=\sup _{U^{\prime} \in B}\left|H / U^{\prime}\right|=|k|$, and thus, according to Proposition A.1 (1), there are $\leq 2^{\max (|k|,|E|)}$ isomorphism classes of cyclic objects of $\mathcal{S} m_{H}(E)$. Let $U$ be the (setwise) stabilizer in $H$ of an algebraically closed subfield $F^{\prime} \neq k$ in $F \mid k$ of a finite transcendence degree.

Note, that if the $H$-action on $E$ is trivial then there are

- $\left|\operatorname{Hom}\left(\mathbb{Q}_{+}^{\times}, E^{\times}\right)\right|=\max \left(2^{|\mathbb{N}|},|E|\right)$ one-dimensional representations of $U$, that factor through the composition of the surjective continuous homomorphisms $U \longrightarrow G_{F^{\prime} \mid k} \stackrel{\chi}{\longrightarrow} \mathbb{Q}_{+}^{\times}$, where $\chi$ is the modulus of $G_{F^{\prime} \mid k}$;

- $\geq|k|$ smooth irreducible $E$-representations of $H$ : for each elliptic curve $A$ over $k$ without complex multiplication the smooth representation $(A(F) / A(k)) \otimes E$ of $H$ is irreducible.

By Proposition A.1 (3), there are exactly $2^{|\mathbb{N}|}$ smooth irreducible $E$-representations of $G$, if $k$ and $E$ are countable.

Proposition A.1 shows, there are 'too many' $\left(\geq \max \left(2^{|\mathbb{N}|},|k|,|E|\right)\right)$ smooth irreducible representations of $G$. (This is one of the reasons to study rather $\mathcal{I}_{G}$ than $\mathcal{S} m_{G}(E)$, where the objects are supposed to be more controllable, since it is expected that they are of 'cohomological nature'.)

2 . If $H$ is locally compact, but not unimodular, then there are $\geq \max \left(2^{\mathrm{rk}\left(\chi_{H}(H)\right)},|E|\right)$ irreducible representations. E.g., if $H$ is the automorphism group of a non-trivial algebraically closed field extension of $k$ of finite transcendence degree, we get the bound $\geq \max \left(2^{|\mathbb{N}|},|E|\right)$.

A.6. Injectives in $\mathcal{S}_{H}(E)$ and in $\mathcal{I}_{G}$. For any $E$-vector space $V$, let $\operatorname{Maps}(H, V)$ be the module of $V$-valued functions on $H$. We endow $\operatorname{Maps}(H, V)$ with the following $E$-vector space structure: $(\lambda f)(x)=x \lambda \cdot f(x)$ for any $\lambda \in E$. The $H$-module structure on $\operatorname{Maps}(H, V)$ is given by the right translations of the argument $h: f(x) \mapsto f(x h)$ for any $h \in H$.

Define $I(V)$ as the smooth part of $\operatorname{Maps}(H, V)$, i.e., $I(V):=\lim _{U^{\prime} \in B} \operatorname{Maps}\left(H / U^{\prime}, V\right)$. Clearly, $I(V)$ is a $E$-vector subspace of $\operatorname{Maps}(H, V)$ invariant under the $H$-action. More generally, for any subgroup $U \subset H$ denote by $\operatorname{Ind}_{U}^{H}: \mathcal{S} m_{U}(E) \longrightarrow \mathcal{S} m_{H}(E)$ the smooth induction functor $V \longmapsto \underset{U^{\prime} \in B}{\lim _{U}} \operatorname{Maps}_{U}\left(H / U^{\prime}, V\right)$, where $\operatorname{Maps}_{U}\left(H / U^{\prime}, V\right)$ is the group of $U$-equivariant maps.

In fact, $\operatorname{Ind}_{U}^{H}$ is right adjoint to the forgetful functor $\operatorname{Res}_{U}^{H}: \mathcal{S} m_{H}(E) \longrightarrow \mathcal{S} m_{U}(E)$ :

$$
\operatorname{Hom}_{\mathcal{S} m_{H}(E)}\left(A, \operatorname{Ind}_{U}^{H}(V)\right)=\operatorname{Hom}_{E[H]}\left(A, \operatorname{Maps}_{U}(H, V)\right)=\operatorname{Hom}_{\mathcal{S} m_{U}(E)}\left(\operatorname{Res}_{U}^{H} A, V\right)
$$


for any $A \in \mathcal{S} m_{H}(E)$ and $V \in \mathcal{S} m_{U}(E)$, where $\operatorname{Hom}_{E[H]}\left(A, \operatorname{Maps}_{U}(H, V)\right) \ni \varphi \mapsto(a \mapsto \varphi(a)(1)) \in$ $\operatorname{Hom}_{E[U]}(A, V) ; \operatorname{Hom}_{E[U]}(A, V) \ni \psi \mapsto(a \mapsto(h \mapsto \psi(h a))) \in \operatorname{Hom}_{E[H]}\left(A, \operatorname{Maps}_{U}(H, V)\right)$.

Therefore, if $V$ is an injective object in $\mathcal{S} m_{U}(E)$ then $\operatorname{Ind}_{U}^{H}(V)$ is an injective object in $\mathcal{S} m_{H}(E)$. In particular, it follows from the semi-simplicity of the category of $E$-vector spaces that its arbitrary object is injective, and thus, $I(V)=\operatorname{Ind}_{\{1\}}^{H}(V)$ is also injective. If, moreover, $V$ is a smooth $H$ module then $V \longrightarrow I(V), v \mapsto(h \mapsto h v)$, is a monomorphism in $\mathcal{S}_{H}(E)$. This means that there are enough injectives in $\mathcal{S} m_{H}(E)$.

Lemma A.2 (Mi] Ch.III, Lemma 1.3). If in an abelian category there are direct sums, products, a generating family and all filtered direct limits are exact then there are enough injectives.

For a subgroup $U$ of $H$ denote by $H_{\mathcal{S} m_{H}}^{q}(U,-)$ the $q$-th derived functor 5 of $H^{0}(U,-)$ on $\mathcal{S} m_{H}$. Evidently, $H_{\mathcal{S} m_{H}}^{q}(U,-)=H_{\mathcal{S} m_{H}}^{q}(\bar{U},-)$, where $\bar{U}$ is the closure of $U$ in $H$.

In other words, $H_{\mathcal{S} m_{H}}^{j}(H,-)=\operatorname{Ext}_{\mathcal{S} m_{H}}^{j}(\mathbb{Q},-)$ can be defined using the smooth cochains.

Lemma A.3. There is a morphism of functors $H_{\mathcal{S} m_{H}}^{*}(U,-) \longrightarrow H_{\mathcal{S} m_{U}}^{*}(U,-)$ on $\mathcal{S} m_{H}$, which is an isomorphism if $U$ is open.

Proof. As the right $\mathbb{Z}[U]$-module $\mathbb{Z}[H]$ is free, the co-induction functor $\mathbb{Z}[H] \otimes_{\mathbb{Z}[U]}-: \mathcal{S} m_{U} \longrightarrow$ $\mathcal{S} m_{H}$ is exact. On the other hand, it is left adjoint to the forgetful functor $\operatorname{Res}_{U}^{H}: \mathcal{S} m_{H} \longrightarrow \mathcal{S} m_{U}$, and therefore, for any injective object $I \in \mathcal{S} m_{H}$ the functor $\operatorname{Hom}_{U}\left(-, \operatorname{Res}_{U}^{H}(I)\right)=\operatorname{Hom}_{H}\left(\mathbb{Z}[H] \otimes_{\mathbb{Z}[U]}\right.$ $-, I)$ on $\mathcal{S} m_{U}^{\text {op }}$ is exact. In other words, the forgetful functor $\operatorname{Res}_{U}^{H}$ transforms injectives of $\mathcal{S} m_{H}$ to injectives of $\mathcal{S} m_{U}$, and thus, an injective resolution of $W$ in $\mathcal{S} m_{H}$ becomes an injective resolution of $\operatorname{Res}_{U}^{H}(W)$ in $\mathcal{S} m_{U}$.

REMARK. Evidently, the morphism of functors $H_{\mathcal{S} m_{H}}^{*}(U,-) \longrightarrow H_{\mathcal{S} m_{U}}^{*}(U,-)$ on $\mathcal{S} m_{H}$, is not an isomorphism for arbitrary $U$ : if $H$ is topologically simple, e.g. $H=G$, and $U$ is locally compact and not unimodular, e.g. $U=G_{F \mid F^{\prime}}$ for a subfield $F^{\prime} \subset F$, over which $F$ is of finite transcendence degree, then $H_{\mathcal{S} m_{H}}^{*}(U, \mathbb{Q})=0 \neq H_{\mathcal{S} m_{U}}^{*}(U, \mathbb{Q})$.

Lemma A.4. Let $U \subset H$ be a subgroup, and $\left\{U \subset U_{\alpha} \subset H\right\}_{\alpha}$ be a partially ordered collection of subgroups, which is filtering for the neighbourhoods of $[1] \in H / \bar{U}$, i.e. any neighbourhood of $[1] \in H / \bar{U}$ contains $U_{\alpha} / U_{\alpha} \cap \bar{U} \subset H / \bar{U}$ for some $\alpha$. Then $\lim _{\alpha} H_{\mathcal{S} m_{H}}^{q}\left(U_{\alpha}, V\right) \stackrel{\sim}{\longrightarrow} H_{\mathcal{S} m_{H}}^{q}(U, V)$.

Proof. Let $I^{\bullet}(V)$ be an injective resolution of $V$. By definition, $H_{\mathcal{S} m_{H}}^{q}(U, V)=H^{q}\left(I^{\bullet}(V)^{U}\right)$. As all $I^{i}(V)$ are smooth, $I^{\bullet}(V)^{U}=\lim _{\alpha} I^{\bullet}(V)^{U_{\alpha}}$. As the direct limits commute with the cohomology, $H^{q}\left(\lim _{\alpha} I^{\bullet}(V)^{U_{\alpha}}\right)=\lim _{\alpha} H^{q}\left(I^{\bullet}(V)^{U_{\alpha}}\right)=\lim _{\alpha} H_{\mathcal{S} m_{H}}^{q}\left(U_{\alpha}, W\right)$, which completes the proof.

Example. For any subgroup $U$ of $H$ the collection $\left\{\left\langle U, U^{\prime}\right\rangle\right\}_{U^{\prime} \in B}$ of subgroups of $H$, generated by $U$ and the elements of $B$, satisfies the assumptions of Lemma A.4.

In this case $\lim _{\alpha} H_{\mathcal{S} m_{U_{\alpha}}}^{q}\left(U_{\alpha}, W\right) \stackrel{\sim}{\longrightarrow} H_{\mathcal{S} m_{H}}^{q}(U, W)$ by Lemma A.3.

Proposition A.5. For any $j \geq 0$ the restriction to $\mathcal{S}_{H}(E)$ of the functor $\operatorname{Ext}_{\mathcal{S} m_{H}}^{j}(\mathbb{Q},-)$ coincides with $\operatorname{Ext}_{\mathcal{S} m_{H}(E)}^{j}(E,-)$. Clearly, $H^{0}(H,-)=\operatorname{Hom}_{H}(\mathbb{Q},-)=\operatorname{Hom}_{\mathcal{S}_{H}(E)}(E,-)$ on $\mathcal{S}_{H}(E)$.

Proof. This is a particular case of [SGA 4 II, Exposé V, Corollaire 3.5] and follows from the fact that the objects $W$ of $\mathcal{S} m_{H}(E)$ admit canonical injective resolutions $I(W) \rightarrow I(I(W) / W) \rightarrow$

\footnotetext{
${ }^{5}$ As there are enough injectives in $\mathcal{S} m_{H}$ the derived functors of left exact functors are defined.
} 
$I(I(I(W) / W) / I(W)) \rightarrow \ldots$ such that the forgetful functor $\mathcal{S} m_{H}(E) \longrightarrow \mathcal{S} m_{H}$ transforms them to injective resolutions in $\mathcal{S} m_{H}$. The injectivity follows from the above adjointness property (3).

Corollary A.6. There are enough injectives in $\mathcal{S m}_{H}(E)$ and in $\mathcal{I}_{G}$.

Proof. Direct sums and direct products in $\mathcal{S}_{H}(E)$ and in $\mathcal{I}_{G}$ were already defined in Appendix A.2. Direct limits in all these categories are direct limits in the category of abelian groups, and therefore, they are exact. For $\mathcal{S}_{H}(E)$ the family $\left(A_{U}=E[H / U]\right)_{U \in B}$ is generating. (Clearly, the direct sum or direct product of all objects in a generating family of $\mathcal{S} m_{H}(E)$ is a generator.) In the case $E=F$ for each positive integer $m$ fix a field extension $K_{m}$ of $k$ of a finite transcendence degree $\geq m$. Then $\left(A_{m}=F\left[G / G_{F \mid K_{m}}\right]\right)_{m \geq 1}$ is another generating family for $\mathcal{S}_{G}(F)$. The functor $\mathcal{I}$, left adjoint to the inclusion $\mathcal{I}_{G} \hookrightarrow \mathcal{S} m_{G}$, cf. [R1], transforms any generating family for $\mathcal{S} m_{G}$ to a generating family for $\mathcal{I}_{G}$.

A.7. Projectives in $\mathcal{S} m_{H}(E)$. Recall, cf. p 12, that $\widehat{H}:=\lim _{U} H / U$ is a semi-group.

Lemma A.7. The following conditions are equivalent:

(1) there is a locally compact group $H^{\prime}$ and an equivalence of categories $\mathcal{S} m_{H}(E) \stackrel{\sim}{\longrightarrow} \mathcal{S} m_{H^{\prime}}(E)$;

(2) $\widehat{H}$ is a locally compact group, so $\widehat{H}=\lim _{U} U \backslash H$, and $H \longrightarrow \widehat{H}$ is an embedding with dense image such that the topology of $H$ is induced by the topology of $\widehat{H}$;

(3) there exists an open subgroup of $H$ such that its any open subgroup is of finite index;

(4) there exists a non-zero projective object in the category $\mathcal{S m}_{H}(E)$ for some $E$;

(5) there exist enough projective objects in the category $\mathcal{S m}_{H}(E)$ for any $E$.

Proof. It is evident that (2) and (3) are equivalent. The implication (5) $\Rightarrow(4)$ is trivial.

$(3) \Rightarrow(5)$. Let $U$ be an open subgroup of $H$ such that its any open subgroup is of finite index. Then the objects $E\left[H / U^{\prime}\right]$ are projective for all open subgroups $U^{\prime}$ of $U$ and form a system of generators of $\mathcal{S}_{G}(E)$.

(44) $\Rightarrow(3)$. Let $W \in \mathcal{S} m_{H}(E)$ be a projective object. Choose a generating system $\left\{e_{j}\right\}_{j \in J}$ of the representation $W$. This gives rise to a surjective homomorphism $\bigoplus_{j \in J} E\left[H / \mathrm{Stab}_{e_{j}}\right] \stackrel{\pi}{\longrightarrow} W$. Fix an element $i_{0} \in J$ and for each $j \in J$ fix an open subgroup $U_{j}$ in $\operatorname{Stab}_{e_{j}} \cap \mathrm{Stab}_{e_{i_{0}}}$. As $W$ is projective, the composition of $\pi$ with the surjection $\bigoplus_{j \in J} E\left[H / U_{j}\right] \longrightarrow \bigoplus_{j \in J} E\left[H / \mathrm{Stab}_{e_{j}}\right]$ splits, and therefore, there exists an element in $\bigoplus_{j \in J} E\left[H / U_{j}\right]$ with the same stabilizer as $e_{i_{0}}$. This implies that the space $E\left[H / U_{j}\right]^{\operatorname{Stab}_{e_{i}}}$ is non-zero for some $j$, and thus, the index of $U_{j}$ in $\operatorname{Stab}_{e_{i}}$ is finite. In other words, any open subgroup of $\mathrm{Stab}_{e_{i_{0}}}$ is of finite index.

EXAMPLES. 1. In the case $H=G$ any open subgroup of $H$ contains an open subgroup of infinite index, and thus, there are no non-zero projective objects in the category $\mathcal{S}_{G}(E)$.

2. If $H$ is locally compact then there are enough projectives in $\mathcal{S}_{H}(E)$.

A.8. Left exact subfunctors of the forgetful functor from $\mathcal{S} m_{H}(E)$ to the category of $E$-vector spaces. The functors $H^{0}(U,-)$ on $\mathcal{S}_{H}(E)$ for subgroup $6 \subseteq \subseteq H$ are examples of left exact subfunctors of the forgetful functor from $\mathcal{S} m_{H}(E)$ to the category of $E$-vector spaces.

Fix a 'sufficiently big' $E$-vector space $W_{0}$. If $\operatorname{dim}_{E} W_{0} \geqslant|H / U|$ for any open subgroup $U \subset H$ then any finitely generated representation of $H$ is embeddable into $I\left(W_{0}\right)$.

\footnotetext{
${ }^{6}$ Clearly, $H^{0}(U,-)=H^{0}\left(U^{\prime},-\right)$, where $U^{\prime}$ is the intersection of all open subgroups in $H$ containing $U$.
} 
Proposition A.8. There is a natural bijection between the E-vector subspaces in $I\left(W_{0}\right)$, invariant under the algebra $\operatorname{End}_{E[H]}\left(I\left(W_{0}\right)\right)=\operatorname{Hom}_{E}\left(I\left(W_{0}\right), W_{0}\right)$, and left exact subfunctors of the forgetful functor from $\mathcal{S m}_{H}(E)$ to the category of E-vector spaces.

Namely, any functor $\Phi$ is sent to the subspace $\Phi\left(I\left(W_{0}\right)\right)$. Conversely, as the $E$-vector space $W_{0}$ is 'big enough', any 'sufficiently small' object of $\mathcal{S} m_{H}(E)$ is embeddable into $I\left(W_{0}\right)$, and thus, one can define $\Phi(W):=W \bigcap \Phi\left(I\left(W_{0}\right)\right)$, which is independent of the choice of the embedding.

\section{ApPendix B. Non-DEgenerate GENERICALly FINITE CORRESPONDENCES AND SMOOTH $G$-MODULES COINDUCED FROM OPEN SUBGROUPS}

Consider the category of smooth $k$-varieties with the morphisms, given by formal linear combinations of non-degenerate generically finite correspondences, i.e. irreducible subvarieties in the product of the source and the target, generically finite over a connected component of the source and dominant over a connected component of the target: $\operatorname{Hom}(X, Y)=Z^{\operatorname{dim} Y}\left(k(X) \otimes_{k} k(Y)\right)$ for connected $X$ and $Y$.

Then there is a full embedding of this category into the category of smooth representations of $G$, given by $X \mapsto Z^{\operatorname{dim} X}\left(k(X) \otimes_{k} F\right)=\mathbb{Q}[\{k(X) \stackrel{/ k}{\hookrightarrow} F\}]$. If we fix a $k$-field embedding of the function field $k(X)$ into $F$ then the module $Z^{\operatorname{dim} X}\left(k(X) \otimes_{k} F\right)$ of generic 0-cycles on $X_{F}$ becomes $\mathbb{Q}\left[G / G_{F \mid k(X)}\right]$. These $G$-modules are very complicated.

One can extract dimension of $X$ out of $W=Z^{\operatorname{dim} X}\left(k(X) \otimes_{k} F\right)$ : it is equal to the minimal $q \geq 0$ such that $W^{G_{F \mid \bar{L}}} \neq 0$, where $\operatorname{tr} \cdot \operatorname{deg}(L \mid k)=q$. Also, 'birational motivic' invariants 'modulo isogenies', such as $\operatorname{Alb}(X), \Gamma\left(X, \Omega_{X \mid k}^{\bullet}\right)=\operatorname{Hom}_{G}\left(C_{k(X)}, \Omega_{F \mid k}^{\bullet}\right)$, can be recovered from $W$, cf. [R1, R2. However, we do not know, whether the birational type of $X$ is determined by $W$.

The collection $\mathrm{JH}(X)$ of irreducible subquotients of the $G$-module of generic 0-cycles on $X$ over $F$ is a birational invariant of $X$. In this section we give examples of pairs of non-birational varieties $X$ and $Y$, with the same collections $\mathrm{JH}(X)$ and $\mathrm{JH}(Y)$. It is not even excluded that $\mathrm{JH}(X)$ depends only on $\operatorname{dim} X$ (and moreover, it is not even shown yet that it does depend on $X \neq \operatorname{Spec}(k)$ ).

To show the inclusion $\mathrm{JH}(X) \supseteq \mathrm{JH}(Y)$, it suffices to construct a $G$-embedding of $Z^{\operatorname{dim} X}\left(k(Y) \otimes_{k}\right.$ $F$ ) into a cartesian power of $Z^{\operatorname{dim} X}\left(k(X) \otimes_{k} F\right)$. For any generically finite map $X \longrightarrow Y$ the pullback induces a desired embedding $Z^{\operatorname{dim} X}\left(k(Y) \otimes_{k} F\right) \hookrightarrow Z^{\operatorname{dim} X}\left(k(X) \otimes_{k} F\right)$. (This is a particular case of the following situation. Let $U \subset U^{\prime} \subset H$ be subgroups, and let index of $U$ in $U^{\prime}$ be finite. Then $[u] \mapsto \sum_{h \in H / U, h U^{\prime}=u U^{\prime}}[h]$ gives a natural $H$-embedding $E\left[H / U^{\prime}\right] \hookrightarrow E[H / U]$.)

Proposition B.1 ([R1], Corollary 7.3). Let $Z$ be a k-variety, $Z^{\prime}$ be a generically twofold cover of $Z$, $X=Z \times \mathbb{P}^{1}$ and $Y=Z^{\prime} \times \mathbb{P}^{1}$. Then there are $G$-embeddings $Z^{\operatorname{dim} X}\left(k(X) \otimes_{k} F\right) \hookrightarrow Z^{\operatorname{dim} X}\left(k(Y) \otimes_{k} F\right)$ and $Z^{\operatorname{dim} X}\left(k(Y) \otimes_{k} F\right) \hookrightarrow Z^{\operatorname{dim} X}\left(k(X) \otimes_{k} F\right)$, so $\mathrm{JH}(X)=\mathrm{JH}(Y)$.

This results from the following combinatorial claim ([R1, Lemma 7.2]).

Let $H$ be a group and $U$ and $U^{\prime}$ be subgroups of $H$ such that $U \cap U^{\prime}$ is of index two in $U$ : $U=\left(U \cap U^{\prime}\right) \bigcup \sigma\left(U \cap U^{\prime}\right)$. Suppose that $\tau_{1} \cdots \tau_{N} \neq 1$ for any integer $N \geq 1$ and for any collection $\tau_{1}, \ldots, \tau_{N} \in U^{\prime} \sigma \backslash U$. Then the morphism of E-representations $E[H / U] \stackrel{[\xi] \mapsto[\xi \sigma]+[\xi]}{\longrightarrow} E\left[H / U^{\prime}\right]$ of $H$ is injective.

Proposition B.2 ([R1], 7.4). Fix an odd integer $m \geq 1$, and let the affine $(m-1)$-dimensional $k$-variety $Y$ be given by equation $\sum_{j=1}^{m} x_{j}^{d}=1$, where $d \in\{m+1, m+2\}$. Let $X$ be the quotient of $Y$ by $\left\langle e_{1} e_{2}^{2} \cdots e_{m}^{m}\right\rangle$, where $e_{i} x_{j}=\zeta^{\delta_{i j}} \cdot x_{j}$ for a primitive $d$-th root of unity $\zeta$. Then $\mathrm{JH}(X)=\mathrm{JH}\left(\mathbb{P}_{k}^{m-1}\right)$. 
Proposition B.3. Let $U$ be a subgroup of $H$ and $g_{1}, \ldots, g_{N}$ be involutions in $N_{H} U / U$, generating an infinite subgroup of $N_{H} U / U$. Then the natural map of E-representations $r: E[H / U] \longrightarrow$ $\bigoplus_{j=1}^{N} E\left[H /\left\langle U, g_{j}\right\rangle\right]$ of $H$ is injective.

In particular, if $g_{1}, \ldots, g_{N}$ are rational involutions of a $k$-variety $X$, generating an infinite group, then the natural $G$-morphism $Z^{\operatorname{dim} X}\left(k(X) \otimes_{k} F\right) \longrightarrow \bigoplus_{j=1}^{N} Z^{\operatorname{dim} X}\left(k(X)^{\left\langle g_{j}\right\rangle} \otimes_{k} F\right)$ is injective.

Proof. If a non-zero 0-cycle $\alpha$ is in the kernel of $r$, and $P$ is a point in the support of $\alpha$, then the support of $\alpha$ contains the $\left\langle g_{1}, \ldots, g_{N}\right\rangle$-orbit of the point $P$. As this orbit is infinite, but the support of $\alpha$ is finite, we get the contradiction, i.e., $\alpha=0$.

EXAMPLES. 1. Let $X$ be an algebraic $k$-group, $g_{1}: x \mapsto x^{-1}$ and $g_{2}: x \mapsto h \cdot x^{-1}$, where $h \in X(k)$ is a point of infinite order. Then the $E$-representations $E[\{k(X) \stackrel{/ k}{\hookrightarrow} F\}]$ and $E[\{k(K(X)) \stackrel{/ k}{\hookrightarrow} F\}]$ of $G$ have the same irreducible subquotients, where $K(X)$ is the quotient of $X$ by the involution $g_{1}$ (the Kummer variety).

2. Let $X=\prod_{j=1}^{N} Y_{j}$ be a product of generically twofold covers $Y_{j}$ of projective spaces (e.g., hyperelliptic curves) over $k$, at least one of which, for example $Y_{1}$, is a curve of genus $\leq 1$. Then there are embeddings of $G$-representations $Z^{d}\left(k(X) \otimes_{k} F\right) \hookrightarrow \bigoplus_{i=1}^{N} Z^{d}\left(k\left(\prod_{1 \leq j \leq N, j \neq i} Y_{j}\right)\left(\mathbb{P}^{d_{i}}\right) \otimes_{k}\right.$ $F)^{1+\delta_{1 i} \hookrightarrow} Z^{d}\left(k\left(\mathbb{P}^{d}\right) \otimes_{k} F\right)^{N+1}$, where $d_{i}=\operatorname{dim} Y_{i}$ and $d=\operatorname{dim} X$. In particular, $\operatorname{JH}(X)=\mathrm{JH}\left(\mathbb{P}_{k}^{d}\right)$.

\section{Appendix C. Differential forms and Cohomologies}

For any field extension $L \mid k$ let $H_{\mathrm{dR} / k, c}^{q}(L)$ be the image in $H_{\mathrm{dR} / k}^{q}(L):=\operatorname{coker}\left[\Omega_{L \mid k}^{q-1} \stackrel{d}{\longrightarrow} \Omega_{L \mid k \text {,closed }}^{q}\right]$ of $\lim H_{\mathrm{dR} / k}^{q}(X)$, where $X$ runs over smooth proper models of subfields in $L$ of finite type over $k$. If $L$ is algebraically closed, this is an admissible representation of $G_{L \mid k}$ over $k$, cf. the footnote on $\mathrm{p}$.9. This is a remarkable object and its structure reflects several, so far conjectural, relations between the cohomology and algebraic cycles. E.g., when $k=\mathbb{C}$ the Hodge general conjecture is equivalent to the vanishing of any Hodge substructure in $H_{\text {sing, } c}^{q}(L)$ with $h^{q, 0}=0$. In other words, it is equivalent to the vanishing of the subrepresentation $\mathfrak{N}^{1} H_{\text {sing,c }}^{q}(F)$, which is by definition the maximal Hodge substructure in $H_{\text {sing, } c}^{q}(F)$ with $h^{q, 0}=0$.

The Hodge filtration on $\Omega_{X \mid k}^{\bullet}$ induces a descending filtration on $H_{\mathrm{dR} / k, c}^{q}(L)$ with the graded quotients $H_{L \mid k}^{p, q-p}=\underline{\lim } \operatorname{coker}\left[H^{p-1}\left(D, \Omega_{D \mid k}^{q-p-1}\right) \longrightarrow H^{p}\left(X, \Omega_{X \mid k}^{q-p}\right)\right]$, where $(X, D)$ runs over the pairs consisting of a smooth proper variety $X$ with $k(X) \subset L$ and a normal crossing divisor $D$ on $X$ with smooth irreducible components. More particularly, $H_{L \mid k}^{q, 0}=\Omega_{L \mid k, \text { reg }}^{q} \subset H_{\mathrm{dR} / k, c}^{q}(L)$.

It is easy to see that the restrictions of $\Omega_{F \mid k, \text { reg }}^{q}$ and of $H_{\text {sing,c }}^{q}(F) / \mathfrak{N}^{1}$ to any compact subgroup $U$ of $G$ contain the same irreducible representations of $U$. Indeed, any finite group $\Gamma$ acting on a smooth proper complex variety $X$ induces automorphisms of the Hodge structure $H_{\text {sing }}^{q}(X)$. For any irreducible representation $\rho$ of $\Gamma$ the element $p_{\rho}=\frac{\operatorname{deg} \rho}{|\Gamma|} \sum_{\gamma \in \Gamma} \operatorname{tr} \rho\left(\gamma^{-1}\right) \gamma \in \mathbb{Q}[\Gamma]$ is a projector to the $\rho$-isotypical part. Then $p_{\rho} H_{\text {sing }}^{q}(X) \subseteq H_{\text {sing }}^{q}(X)_{\mathbb{Q}}$ is a Hodge substructure, and therefore, $\Gamma\left(X, \Omega_{X}^{q}\right)$ and $H_{\text {sing }}^{q}(X) / \mathfrak{N}^{1}$ contain the same irreducible representations of $\Gamma$.

Proposition. Suppose that the cardinality of $k$ is at most continuum. Fix an embedding $\iota: k \hookrightarrow \mathbb{C}$ into the field of complex numbers. Then

- there is a non-canonical $\mathbb{Q}$-linear isomorphism $H_{F \mid k}^{p, q} \cong H_{F \mid k}^{q, p}$, and a $\mathbb{C}$-anti-linear canonical

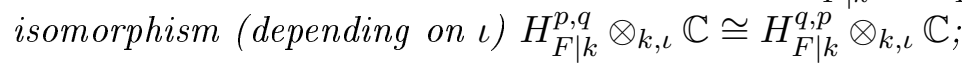

- the representation $H_{\mathrm{dR} / k, c}^{n}(L)$ of $G_{L \mid k}$ is semi-simple for any algebraically closed $L$ of a finite transcendence degree $n$ over $k$ (and in particular, $\Omega_{L \mid k, \text { reg }}^{n}$ is also semi-simple).

Proof. 
- The complexification of the projection $F^{p} H_{\mathrm{dR} / k}^{p+q}(X) \longrightarrow H^{q}\left(X, \Omega_{X \mid k}^{p}\right)$ identifies the space $F^{p} H_{\mathrm{dR} / k}^{p+q}(X) \otimes_{k, \iota} \mathbb{C} \cap \overline{F^{q} H_{\mathrm{dR} / k}^{p+q}(X) \otimes_{k, \iota} \mathbb{C}}$ with $H^{q}\left(X, \Omega_{X \mid k}^{p}\right) \otimes_{k, \iota} \mathbb{C}$, where $F^{\bullet}$ is the Hodge filtration. Then the complex conjugation on $H^{p+q}\left(X_{\iota}(\mathbb{C}), \mathbb{C}\right)=H^{p+q}\left(X_{\iota}(\mathbb{C}), \mathbb{R}\right) \otimes_{\mathbb{R}} \mathbb{C}$ identifies $H^{q}\left(X, \Omega_{X \mid k}^{p}\right) \otimes_{k, \iota} \mathbb{C}$ with $H^{p}\left(X_{\iota}(\mathbb{C}), \Omega_{X_{\iota}(\mathbb{C})}^{q}\right)=H^{p}\left(X, \Omega_{X \mid k}^{q}\right) \otimes_{k, \iota} \mathbb{C}$.

- The semi-simplicity of $H_{\mathrm{dR} / k, c}^{n}(L)$ is equivalent to the semi-simplicity of the representation $\mathbb{C} \otimes_{k, \iota} H_{\mathrm{dR} / k, c}^{n}(L)=\bigoplus_{p+q=n} \mathbb{C} \otimes_{k, \iota} H_{L \mid k}^{p, q}$ of $G_{L \mid k}$. For the latter note that there is a positive definite $G_{L \mid k}$-equivariant hermitian form $\left(\mathbb{C} \otimes_{k, \iota} H_{L \mid k}^{p, q}\right) \otimes_{i d, \mathbb{C}, c}\left(\mathbb{C} \otimes_{k, L} H_{L \mid k}^{p, q}\right) \longrightarrow \mathbb{C}(\chi)$, where $c$ is the complex conjugation and $\chi$ is the modulus of $G_{L \mid k}$, given by $(\omega, \eta)=\int_{X_{\iota}(\mathbb{C})} i^{n^{2}+2 q} \omega \wedge$ $\bar{\eta} \cdot\left[G_{L \mid k(X)}\right]$ for any $\omega, \eta \in H_{\text {prim }}^{p, q}\left(X_{\iota}(\mathbb{C})\right)=\mathbb{C} \otimes_{k, \iota} H_{\text {prim }}^{q}\left(X, \Omega_{X \mid k}^{p}\right) \subset \mathbb{C} \otimes_{k, \iota} H_{L \mid k}^{p, q}$. Here $H_{\text {prim }}^{p, q}\left(X_{\iota}(\mathbb{C})\right)$ denotes the subspace orthogonal to the sum of the images of all Gysin maps $H^{p-1, q-1}(D) \longrightarrow H^{p, q}\left(X_{\iota}(\mathbb{C})\right)$ for all desingularizations $D$ of all divisors on $X_{\iota}(\mathbb{C})$.

Acknowledgement. Both authors were supported in a part by INTAS (grant no. 05-1000008-8118). M.R. gratefully acknowledges the support by the Max-Planck-Institut für Mathematik in Bonn (Fall 2004), Alexander von Humboldt-Stiftung (2005 - 2007), Pierre Deligne 2004 Balzan prize in mathematics (2006-2008), and RFBR (grants 06-01-72550-CNRS-L_a, 07-01-92211-НЦНИЛ_a). Also he is grateful to Regensburg University for the hospitality and to Alexander von Humboldt-Stiftung for making his stay in Regensburg possible.

\section{REFERENCES}

[BZ] I.N.Bernstein, A.V.Zelevinsky, Representations of the group $G L(n, F)$, where $F$ is a local non-Archimedean field. Uspehi Mat. Nauk 31 (1976), no. 3(189), 5-70.

[Joh] P.T.Johnstone, Topos theory. Academic Press, London, New York, San Francisco, 1977.

[Mi] J.S.Milne, Étale cohomology. Princeton Math.Series, 33. Princeton Univ.Press, Princeton, N.J., 1980.

[R1] M.Rovinsky, Motives and admissible representations of automorphism groups of fields. Math. Zeit., 249 (2005), no. 1, 163-221, math.RT/0101170.

[R2] M.Rovinsky, Semilinear representations of PGL, Selecta Math., 11 (2005), 491-522, math.RT/0306333.

[R3] M.Rovinsky, On maximal proper subgroups of field automorphism groups. math.RT/0601028

[R4] M.Rovinsky, Automorphism groups of fields, and their representations, Russian Math. Surveys, 62:6 (2007), 1121-1186.

[SGA 4 I] Théorie des topos et cohomologie étale des schémas. Tome 1 LNM 269, Springer-Verlag 1972. [SGA 4 II] Théorie des topos et cohomologie étale des schémas. Tome 2 LNM 270, Springer-Verlag 1972.

NWF I-Mathematik, Universitat Regensburg, Universitatsstrasse 31, 93053 Regensburg

Independent University of Moscow, 119002 Moscow B.Vlasievsky Per. 11 \& Institute for Information Transmission Problems of Russian Academy of Sciences 